\title{
Numerical simulation of water impact of solid bodies with vertical and oblique entries
}

\author{
H.B. Gu, L. Qian, D.M. Causon, C.G. Mingham \\ Centre for Mathematical Modelling and Flow Analysis \\ School of Computing, Mathematics and Digital Technology \\ The Manchester Metropolitan University, \\ Manchester M1 5GD, UK \\ P. Lin
}

State Key Laboratory of Hydraulics and Mountain River Engineering Sichuan University, Chengdu, China

\begin{abstract}
The flow problem of hydrodynamic impact during water entry of solid objects of various shapes and configurations is simulated by a two-fluid free surface code based on the solution of Navier-Stokes equations (NSE) on a fixed Cartesian grid. In the numerical model the free surface is captured by the level set function, and the partial cell method combined with a local relative velocity approach is applied to the simulation of moving bodies. The code is firstly validated using experimental data and other numerical results in terms of the impact forces and surface pressure distributions for the vertical entry of a semi-circular cylinder and a symmetric wedge. Then configurations of oblique water entry of a wedge are simulated and the predicted free surface profiles during impact are compared with experimental results showing good agreement. Finally, a series of tests involving vertical and oblique water entry of wedges with different heel angles are simulated and the results compared with published numerical results. It is found that the surface pressure distributions and forces predicted by the present model generally agree very well with other numerical results based on potential flow theory. However, as the current model is based on the solution of the NSE, it is more robust and can therefore predict, for example, the formation and separation of the thin flow jets (spray) from surface of the wedge and associated ventilation phenomena for the cases of oblique water entry when the horizontal velocity is dominant. It is also noted that potential flow theory can result in over-estimated negative pressures at the tip of the wedge due to its inherent restriction to non-separated flows.
\end{abstract}

KEY WORDS: Water impact; vertical and oblique water entry; two-phase flow; level set; free-surface; partial cell; local relatively stationary method; moving body.

\section{INTRODUCTION}

Water impact (entry) problems are still receiving extensive attention, especially in ship building, ocean and coastal engineering, and the marine renewable energy sector, although the earliest work can be traced back to nearly a century ago. When a blunt body (such as a sea plane, high speed vessel hull, section of petroleum pipeline) drops into water, water impact and slamming occurs. This is a complex flow problem which involves large free surface deformation, the formation of thin jets (spray), air entrainment as well as flow separation and ventilation. In fact, the shape of solid bodies can be symmetric or asymmetric and may be dropped into water vertically or at an angle. Most investigations of water impact problems have been restricted to the case of purely vertical entry. On the other hand, despite their importance in engineering applications, research into oblique water entry and even free fall of wedge-like objects onto free surfaces have just started to emerge in recent years.

Von Karman (1929) proposed the first analytical solution to deal with the hydrodynamics of a landing seaplane. In his method the added mass concept was introduced, which was adopted later by many researchers in the analytical and asymptotic solution of related flow problems. Based on analytical and experimental results for axially symmetric bodies landing on water Hirano and Miura (1970) improved Von Karman' theory by deriving a formula for calculating the virtual mass of a circular plate. Wagner (1932) described an asymptotic solution and proposed a formula to calculate the instantaneous pressure distribution on a two-dimensional wedge by considering the water entry process as a sequence of impact impulses. By disregarding the negative pressure area of Wagner theory, Toyama (1996) expanded Wagner's theory for water entry of a horizontal cylinder. Mackie 
(1962) was the first to propose a fully linearized solution for the rigid wedge water entry problem. Armand \& Cointe (1987) and Cointe (1991) generalized Wagner's theory to the case of vertical entry of a horizontal circular cylinder and several simplified models for the wedge entry problem have also been proposed by Howison, Ockendon \& Wilson (1991), Fraenkel \& Mcleod (1997), and Mei, Liu \& Yue (1999).

Dobrovol'skaya (1969) proposed an exact nonlinear self-similar solution of the water entry problem for a symmetric wedge entering the free surface vertically with constant velocity. Selfsimilarity in the solutions can be assumed for the initial stage of the water impact process when the entry velocity is large enough to make the influence of gravity negligible, but not high enough to make the acoustic influence relevant. Based on this approach, Fraenkel \& Keady (2004) proved the existence and uniqueness of a similarity solution for all deadrise angles of a wedge. However Dobrovol'skaya's method, which reduces the boundary value problem to a nonlinear singular integral equation, can only be used to solve the water entry problem for the wedges with deadrise angles larger than $30^{\circ}$, owing to a singularity at the jet tip. Greenhow (1987) also encountered this difficulty in a fully nonlinear numerical approach to the flow problem. In order to overcome this problem, Zhao \& Faltinsen (1993) removed the thin jet layer from their computation, assuming that pressure variations within the tip region are small. To avoid the singularity at the jet tip, Wu, Sun \& He (2004) proposed an iterative algorithm in which the free surface boundary conditions are transformed to an integral form and the developed jet is modeled with the shallow water equations. The method was later extended by Xu, Duan \& Wu (2008) for the problem of oblique water entry of an asymmetric wedge.

Strictly, water entry of a wedge is not generally self-similar especially for the free fall of wedges and oblique water entry of symmetric and asymmetric wedges under certain flow conditions so the temporal variable may be expected to remain prominent in the solutions. Thus, more general solutions based on potential flow theory using the boundary element method have also been used for this flow problem. Although it is difficult to discretize the geometry in the boundary element method, many works in the area of water impact have concentrated on an improved implementation of boundary conditions in the interaction region between the solid surface and water free surface (Zhao \& Faltinsen 1993, Lu, He and Wu 2000, Wu 2007, Wu, Sun \& He 2004, Xu, Duan \& Wu 2008 ). Korobkin (1996, 1997, 2006), Korobkin and Peregrine (2000), Korobkin and Khabakhpasheva (2006) have researched extensively matters relating to impact including the role of compressibility, energy distribution aerated water etc.

With the development of computational fluid dynamics (CFD), other alternative but more general methods can also be adopted to simulate water impact and entry problems, especially methods based on the solution of the NSE for free surface flows. The advantage of these methods is that they are generic so that intricate details of the free surface deformation, formation of jet like flows and flow separation can be simulated. The main challenge in solving the NSE for water impact and entry problems is how to locate the interface between water, air and solid surface. There are essentially two approaches for solving the NSE numerically: mesh based methods and meshless methods. Meshless methods include the smoothed particle hydrodynamics (SPH) and moving particle semi-implicit (MPS) methods, and related methods. Shao et al. (2006) presented an incompressible SPH model to investigate wave overtopping at coastal structures. Koshizuka et al. (1995) proposed the MPS method to solve the NSE which can be used for free surface flow problems. Oger at el. (2005) applied the Smooth Particle Hydrodynamics method (SPH) to simulate the free fall of a wedge into water and the results are encouraging.

Mesh based methods for free surface flow problems include the well-known volume of fluid (VOF) method (Hirt \& Nichols 1981, Youngs 1982, Ubbink 1997), the level set method (Osher et al., 1988) and the marker and cell method (MAC) (Harlow \& Welch 1965) to capture or track the free surface together with a NSE solver such as the projection method or well known SIMPLE method. Another 
type of mesh based method is the free surface capturing method proposed initially by Kelecy \& Pletcher (1997) and Pan \& Chang (2000), based on the artificial compressibility method with high resolution Riemann solvers, in which the free surface is treated as a contact discontinuity in the density field. The moving interface is captured automatically as part of the numerical solution along with other flow variables. Qian et al. $(2003,2006)$ and Gao et al. (2007) used this method to study wave run up on a beach, wave overtopping at a vertical wall and fluid interaction with moving bodies. More closely related to the topics discussed in this paper, Pereira at el. (2010) applied the VOF technique with a Navier-Stokes solver to simulate rigid wedge entry into calm water.

In this study, a two-fluid free surface flow solver ( $\mathrm{Gu}$ et al. 2009, 2010) based on a numerical solution of the NSE is applied to the water entry flow problem, employing the fast-marching particle level set method to capture free surfaces combined with a partial cell treatment and the local relative stationary method to simulate the geometric shape of moving bodies (Lin 2007). The model has previously been validated against experimental and numerical data for several free surface flow problems involving wave generation in an empty wave tank and wave interactions with fixed and moving bodies. In this paper, vertical and oblique water entry of solid objects will be simulated using the developed method to show its capability and accuracy in predictions relating to this specific flow problem. A particular focus is to examine the detailed flow structure including free surface profiles and interactions with the body during the process of entry. Features include the formation of the thin jets, overturning and re-connection of free surfaces, flow separations and ventilations, for which, in many instances, methods based on potential theory either fail to predict a solution or give erroneous solutions.

In the following sections the numerical model will be first described. Then, a number of test cases involving vertical and oblique water entry of various symmetric and asymmetric solid bodies are presented and results compared with experimental data and other numerical results where available. Finally some conclusions are drawn.

\section{NUMERICAL METHOD}

\subsection{Basic governing equations}

Here, we are solving the governing flow equations in both air and water separated by a free surface with the partial cell technique adopted to model the geometry. The whole computational domain is divided into fluid cells, solid cells and partial cells. When $\theta=1$ the cell is a fluid cell; $\theta=0$ a solid cell; and for $0<\theta<1$ a partial cell, see figure 1 . With the openness function so defined, the equations of motion for incompressible two-phase flow become

$$
\begin{gathered}
\frac{\partial\left(\theta u_{i}\right)}{\partial x_{i}}=0 \\
\frac{\partial\left(\theta u_{i}\right)}{\partial t}+\theta u_{j} \frac{\partial\left(\theta u_{i}\right)}{\partial x_{j}}=-\frac{\theta}{\rho(\phi)} \frac{\partial p}{\partial x_{i}}+\theta g_{i}+\theta \frac{1}{\rho(\phi)} \frac{\partial \tau_{i j}}{\partial x_{j}}
\end{gathered}
$$

When $\theta=1$ the above equations reduce to the unmodified NSE. Here, $i=1,2$ for $2 \mathrm{D}$ and $i=1,2,3$ for 3D. $u_{i}$ is the velocity component in $i$ direction, $p$ is pressure, $\rho$ is density of air or water, and $\tau_{i j}=2 \mu(\phi) \sigma_{i j}, \sigma_{i j}$ is defined as the rate of deformation tensor. In equation (2) $\phi$ is the level set function defined as a signed distance function that is positive in water and negative in air, (Figure 1). Accordingly, the level set transport equation can be derived as

$$
\frac{\partial \phi}{\partial t}+u_{i} \cdot \frac{\partial \phi}{\partial x_{i}}=0
$$

The zero level set is initialized as the position of the free surface between water and air. From the level set function, the density $\rho(\phi)$ and the viscosity $\mu(\phi)$ are written as

$$
\rho(\phi)=\rho_{a}(1-H(\phi))+\rho_{w} H(\phi)
$$




$$
\mu(\phi)=\mu_{a}(1-H(\phi))+\mu_{w} H(\phi)
$$

where $H(\phi)$ is a Heaviside-type function, $\rho_{w}$ and $\mu_{w}$ are density and viscosity of water respectively and $\rho_{a}$ and $\mu_{a}$ are density and viscosity of air. The Heaviside function is used to define a smooth function which ensures that the density or viscosity vary smoothly from $\rho_{\mathrm{a}}$ to $\rho_{w}$ or from $\mu_{a}$ to $\mu_{w}$ within a narrow band around the interface. There are several choices for the Heaviside function. We adopt the following formula,

$$
H(\phi)=\left\{\begin{array}{lr}
0, & \phi<-1.5 h \\
0.5+\frac{\phi}{3 h}+\frac{1}{2 \pi} \sin \frac{2 \pi \phi}{3 h}, & |\phi| \leq 1.5 h \\
1, & \phi>1.5 h
\end{array}\right.
$$

in which $h$ is the local minimum size of the spatial step.

\subsection{Numerical solution in fluid domain}

In the spatial domain, a staggered-grid system is used within which scalars are defined at cell centres and vectors are defined at the centres of cell faces, see figure 1 . The finite difference method is adopted to discretize spatial derivatives on a non-uniform mesh. When a value is required at a location where it is not defined linear interpolation is used to obtain the value. A two-step projection method is used to solve equations (1) and (2) with a combined upwind and central scheme for the convection terms and a central difference scheme to discretize the diffusion terms (Lin 1998). After the convection and diffusion terms have been calculated the tentative velocities $\tilde{u}_{i}$ are obtained from

$$
\frac{\tilde{\theta u} i_{i}-\theta u_{i}^{n}}{\Delta t}=-\theta u_{j} \frac{\partial\left(\theta u_{i}\right)}{\partial x_{j}}+\theta \frac{1}{\rho(\phi)} \frac{\partial \tau_{i j}}{\partial x_{j}}
$$

In general, the tentative velocities do not satisfy the continuity equation (1). Thus, these must be updated by an iterative pressure correction method using,

$$
\frac{\theta u_{i}^{n+1}-\theta \tilde{u_{i}}}{\Delta t}=-\theta \frac{1}{\rho(\phi)} \frac{\partial p^{n+1}}{\partial x_{i}}+\theta g_{i}
$$

where the $p^{n+1}$ is obtained by solving the Poisson pressure equation,

$$
\frac{\partial}{x_{i}}\left(\theta \frac{1}{\rho(\phi)} \frac{\partial p^{n+1}}{\partial x_{i}}\right)=\frac{1}{\Delta t} \frac{\partial \theta \tilde{u_{i}}}{\partial x_{i}}+\frac{\partial}{\partial x_{i}}\left(\theta g_{i}\right)
$$

Equation (9) is obtained by enforcing equation (8) at the time step $n+1, \partial\left(\theta u_{i}^{n+1}\right) / \partial x_{i}=0$, ensures the velocities calculated from equation (8) are divergence free. 


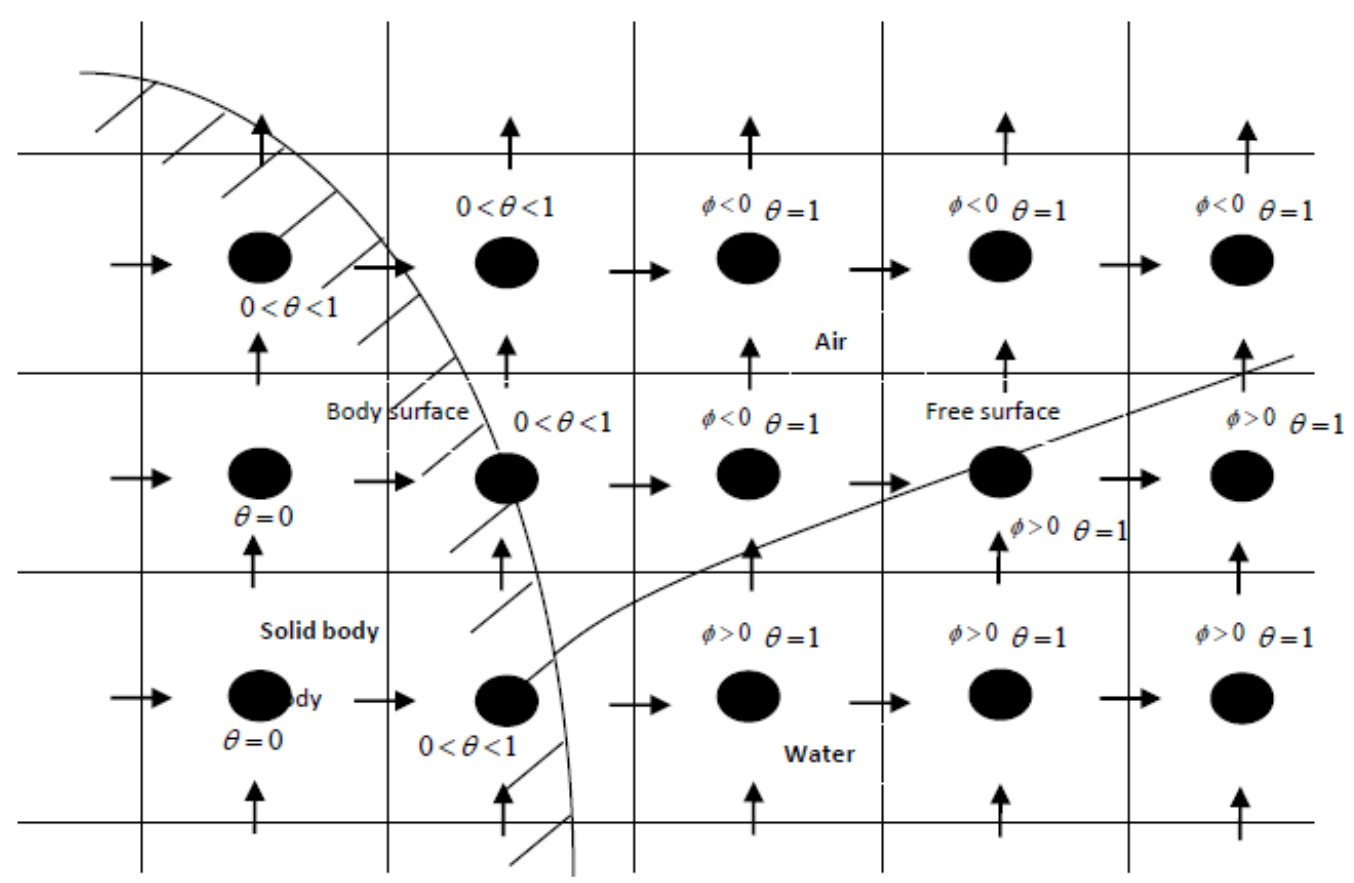

(Velocity stored at arrows and pressure stored at central dots)

Fig.1 Illustration of partial cell technique, level set definition and the storage location of the dependent variables

\subsection{Local relative stationary (LRS) method for a moving boundary}

There are two major issues that require special attention in the numerical implementation of the partial cell method for a moving body. One is tracking the moving body and the other is ensuring conservation of mass and momentum (Lin 2007).

In this study only rigid bodies are considered but the procedures are generalisable. The body surface is expressed by the zero set of a combination of quadratic functions e.g. $F_{m}\left(x, y, t^{n}\right)=0$. The updated position of the body surface on the grid can be determined by simple geometric principles at time step $n+1$, e.g. $F_{m}\left(x, y, t^{n+1}\right)=0$. The openness function $\theta^{n+1}$ can also be updated by a combination of quadratic functions.

To ensure that the conservation laws are satisfied during body movement from one time step to another the following treatment is adopted. We assume that during the small time interval $\Delta t$ the body moves with constant velocities $u_{i B}$, and recast the moving body problem into an equivalent problem where the body remains stationary and the fluid motion is superimposed through an additional velocity with the same magnitude as that of the moving body but in the opposite direction. This is referred to as the local relative stationary method. Details can be found in the paper by Lin (2007). Thus, the continuity equation (1) in the partial cell is revised to be,

$$
\frac{\partial\left(\theta u_{i}-\theta u_{i B}\right)}{\partial x_{i}}=0 \quad \Rightarrow \quad \frac{\partial\left(\theta u_{i}\right)}{\partial x_{i}}=\frac{\partial\left(\theta u_{i B}\right)}{\partial x_{i}}
$$

Substituting the above modified continuity equation into the two-step method, a modified Poisson pressure equation is obtained, 


$$
\frac{\partial}{x_{i}}\left(\theta^{n+1} \frac{1}{\rho(\phi)} \frac{\partial p^{n+1}}{\partial x_{i}}\right)=\frac{1}{\Delta t} \frac{\partial \theta^{n+1}\left(\tilde{\left.u_{i}-u_{i B}\right)}\right)}{\partial x_{i}}+\frac{\partial}{\partial x_{i}}\left(\theta g_{i}\right)
$$

It can be seen that a moving body changes the pressure field around the body, which consequently affects the flow around it. Apart from the pressure field, the moving body affects the viscous stresses. This is considered in the first step of the projection method where the convection and diffusion terms are evaluated. The local relative stationary method is still applied to calculate the relative velocity gradients near the body. Additional terms result that modify the normal and shear stress around the moving body.

The advantages of the local relative stationary method are that it is very easy to implement numerically and easy to deal with any number of multiple bodies since a moving coordinate system is established at the level of an individual cell. No re-meshing is required and the flow computation remains based on a fixed-grid system. The movement of the body is updated in a Lagrangian manner and thus is free of numerical diffusion. Extensions to three-dimensions and more complicated body motion than shown here e.g. with rotation are also straightforward (Lin 2007).

\subsection{Level set method}

There are many methods (Shu at el. 1989, Enright 2005, 2002, Jiang 1999, 2000, Strain 1999, 2000, Mulder 1992) to solve the level set equation (3), amongst these the semi-Lagrangian method has generally high computational efficiency. We modify the original formula to be found in the cited source references for use on non-uniform grids. The distance function in $2 \mathrm{D}$ at the time step $n+1$ can be expressed as,

$$
\phi_{i, j}^{n+1}=\alpha \beta \phi_{r+1, s+1}^{n}+(1-\alpha) \beta \phi_{r, s+1}^{n}+\alpha(1-\beta) \phi_{r+1, s}^{n}+(1-\alpha)(1-\beta) \phi_{r, s}^{n}
$$

where $r$ and $s$ relate to the left bottom corner index of the grid cell and the new grid point value arises from that at the time step $n$ according to the velocities $u_{i, j}$ and $v_{i, j}$. Parameters $\alpha$ and $\beta$ are the coefficients in the bilinear interpolation in the $\mathrm{x}$ and $\mathrm{y}$ directions respectively. The formula can easily be extended to $3 \mathrm{D}$. This scheme is unconditionally stable, so the time step is no longer limited by the CFL condition.

In our implementation, we only calculate the distance function within a narrow band around the interface. Thus, at each time step the zero level set must not be allowed to pass out of this narrow band. This places a modest but acceptable restriction on the maximum allowable time step. Using the highly efficient fast marching method (Sethian 1998) we implement a re-initialization process that is also performed in a narrow band around the interface. The narrow band approach makes a worthwhile saving in computational time. To improve the conservation of the level set method, particles are used to assist the level set method to accurately capture the flow characteristics in under-resolved regions.

\subsection{Level set boundary condition for an obstacle}

Within the numerical model, the level set function at the boundary of a fixed or moving body must be carefully treated. Several researchers have described treatments for this problem by considering the angle between the obstacle local surface normal direction and the water surface normal direction (Yang \& Stern 2009) or by interpolation from a nearby cell (Guendelman at el. 2005). In our model the angle between the water surface and the obstacle surface can remain arbitrary consistent with the arbitrary manner in which the water surface comes into contact with the obstacle surface. By 
assuming a constant slope for the free surface near the solid surface, the level set function values at the solid boundary can be approximated. Using the formula for calculating the distance between a point and line, the level set function of a point $m$ with coordinate $\left(x_{m}, y_{m}\right)$ which is the closest point to a boundary point $n$ can be expressed as

$$
\varphi_{m}=a x_{m}+b y_{m}+c
$$

see figure 2 where point $\mathrm{n}$ is a cell center point where the level set function is defined, and the cell is crossed by a body surface. Around the cell only the cell with cell center point $\mathrm{m}$ is fully occupied by fluid and is closest to the free surface. If $\varphi_{m}$ is its level set value and $c$ is a constant, $(a, b)$ is the normal unit vector which can be calculated from $\nabla \varphi /|\nabla \varphi|$, the boundary level set value of point $\mathrm{n}$ with coordinate $\left(x_{n}, y_{n}\right)$ can then be obtained by

$$
\varphi_{n}=a x_{n}+b y_{n}+c
$$

Equations (13) and (14) can be easily extended to a 3D case. The boundary condition for the level set is also confined within the narrow band.

If the body is moving, the boundary level set value outside of the narrow band must also be considered. Outside the narrow band we set the level set value to $+10^{6}$ in water area and $-10^{6}$ in air area. If the moving body is initially located entirely in air, when it moves into the water region, the moving boundary condition for the level set in the narrow band is calculated by (13) and (14); outside the narrow band we set the moving boundary level set value to that of the cell adjacent to it in the fluid area. This method retains the level set value within the body at $+10^{6}$ when it moves into the water region and allows the body to pass through the interface smoothly. If the moving body is initially only located in the water region, when it moves into the air region, the same method is employed.

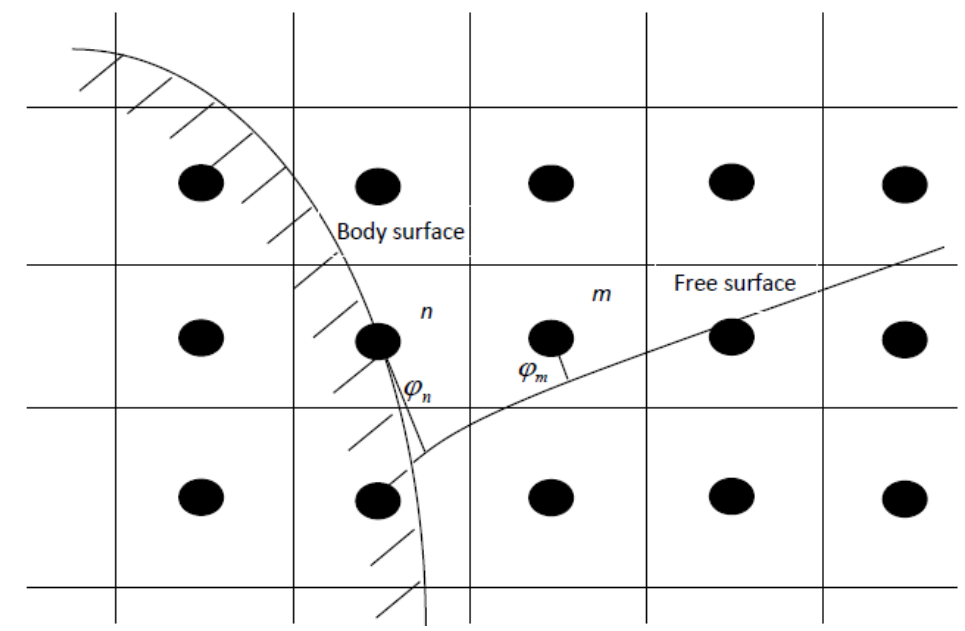

Fig.2 Illustration of body boundary level set condition

\section{NUMERICAL RESULTS}

\subsection{Water entry of a semi-cylinder}

To evaluate the accuracy of the developed numerical model, firstly the vertical water entry of a circular cylinder with a radius of $\mathrm{R}=5.5 \mathrm{~m}$ and a constant vertical velocity of $10 \mathrm{~m} / \mathrm{s}$ is simulated. Figure 3 shows the computational domain and the initial position of the semi-circular cylinder for the test case. The size of the domain is so chosen such that the effects of reflected waves from the domain boundaries can be omitted throughout the simulation. 
In the calculations three levels of refinement of the Cartesian grids and different time steps have been used so that both spatial and temporal convergence of the solutions can be tested. Uniform meshes are used for the area that covers the moving semi-cylinder and non-uniform meshes used in the remaining area of the domain to save computational cost. The minimum spatial intervals of each uniform mesh are $0.2 \mathrm{~m}, 0.1 \mathrm{~m}$ and $0.05 \mathrm{~m}$ respectively. Figure 4 shows the level of mesh convergence. Time convergence is tested on the finest mesh and very similar results were found, as shown by the solid line in figure 4 . In the figure, the force coefficient $\mathrm{Cs}$ is defined as

$$
C_{s}=F /\left(\rho R V^{2}\right)
$$

where $\mathrm{F}$ is the slamming force on the surface of the semi-cylinder, which is calculated by integrating the pressure along its surface, $\rho$ is water density, and $V$ is the dropping velocity.

The results on the finest mesh are compared with various data from theoretical, numerical and experimental work (Seif et al. 2005) as shown in figure 5. There are variations in the predicted Cs values between the theoretical and experimental results. For example, Von Karman's theory gives $C s=\pi$ at initial contact whereas Wagner's modified theory gives $C s=2 \pi$ and the value from the experimental data of Campbell et al. (1980) is at around 5.2. The results from CFD methods including the present method, those of Seif et al. (2005) and Arai et al. (1994) all predict larger impact forces than the experimental data after initial contact. The data for the impact force during the initial contact stage are not shown in the Seif et al. (2005) and Arai et al. (1994) results due to large nonphysical oscillations in the predicted forces. Figure 6 shows the pressure time history at the lowest point on the semi-cylinder predicted by the present model which is very similar to the results obtained by Seif et al. (2005) including the peak pressure values. There is an abrupt pressure increase at the beginning of the impact phase after which the pressure gradually decreases from its peak value. An accurate prediction of the peak pressure values is important to the design of structures particularly as these are difficult to measure accurately in the laboratory. Figure 7 shows the pressure contours at different times during the simulation. At the beginning when the cylinder contacts the calm water a high pressure is present at the contact point but as the cylinder immerses into the water the peak pressure values decrease gradually. The denser contours correspond to higher pressure gradient and indicate that high pressures appear at the spray root. As Seif et al. (2005) conjecture in their paper gravity may have a significant effect on the predicted impact force for this test case. For the present model, with the inclusion of gravity in the calculations the predicted slamming force coefficients are approximately $20 \%$ higher than those without gravity. However, the peak value of the slamming force appears to be less affected by gravity.

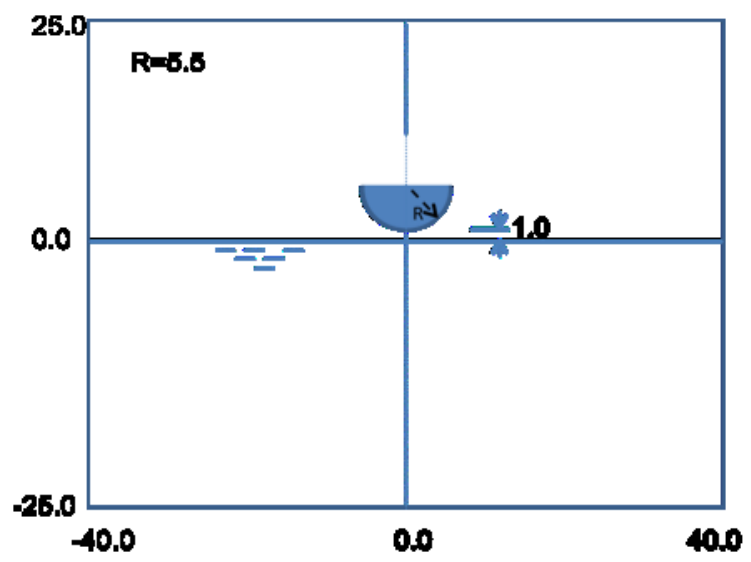

Fig.3 Size of the computational domain for the water entry of a semi-cylinder 


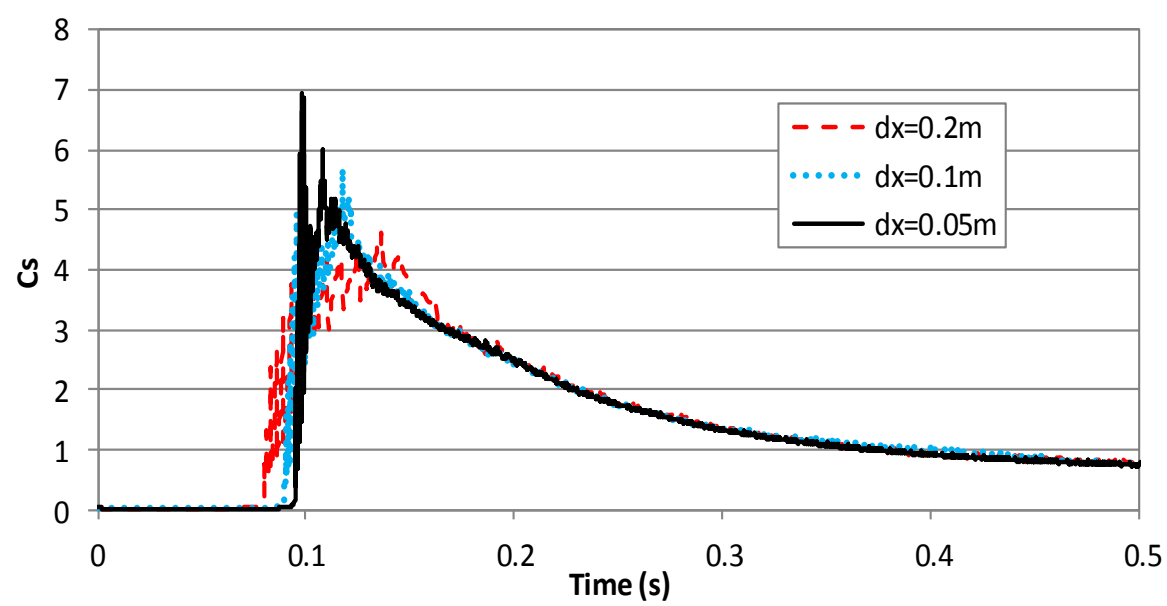

Fig.4 Grid convergence test: slamming force coefficient results

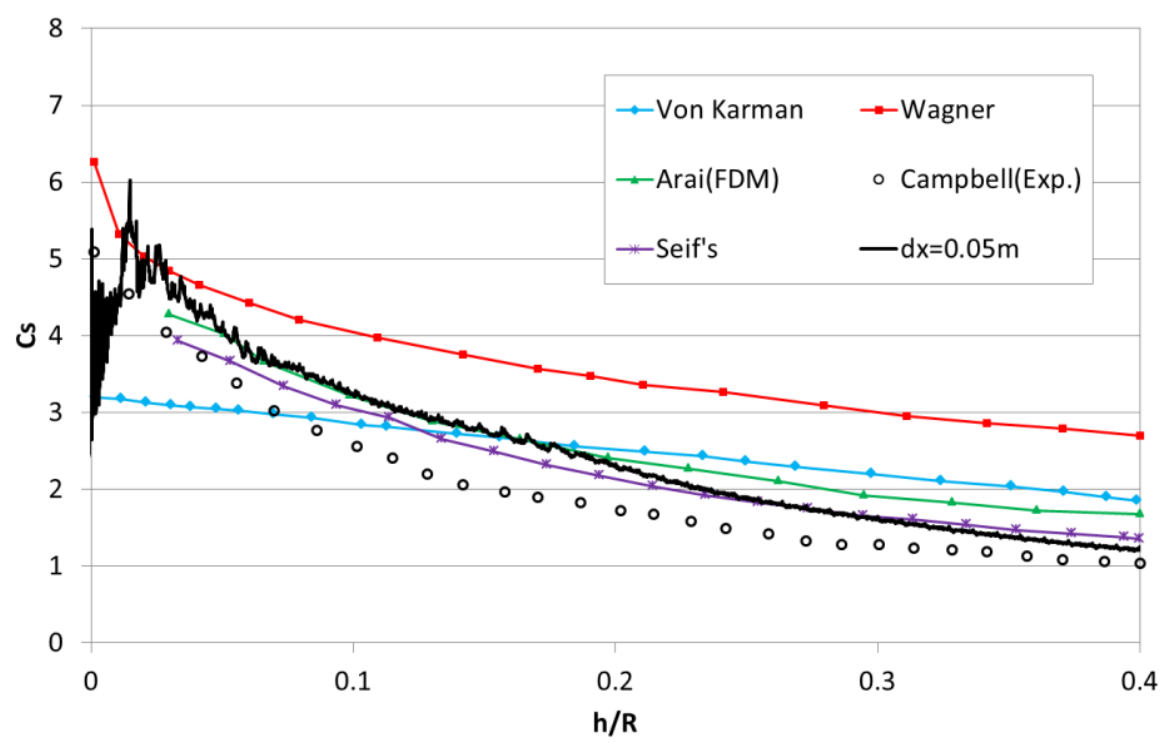

Fig.5 Comparison of slamming force coefficient over $\mathrm{h} / \mathrm{R}$ with other data

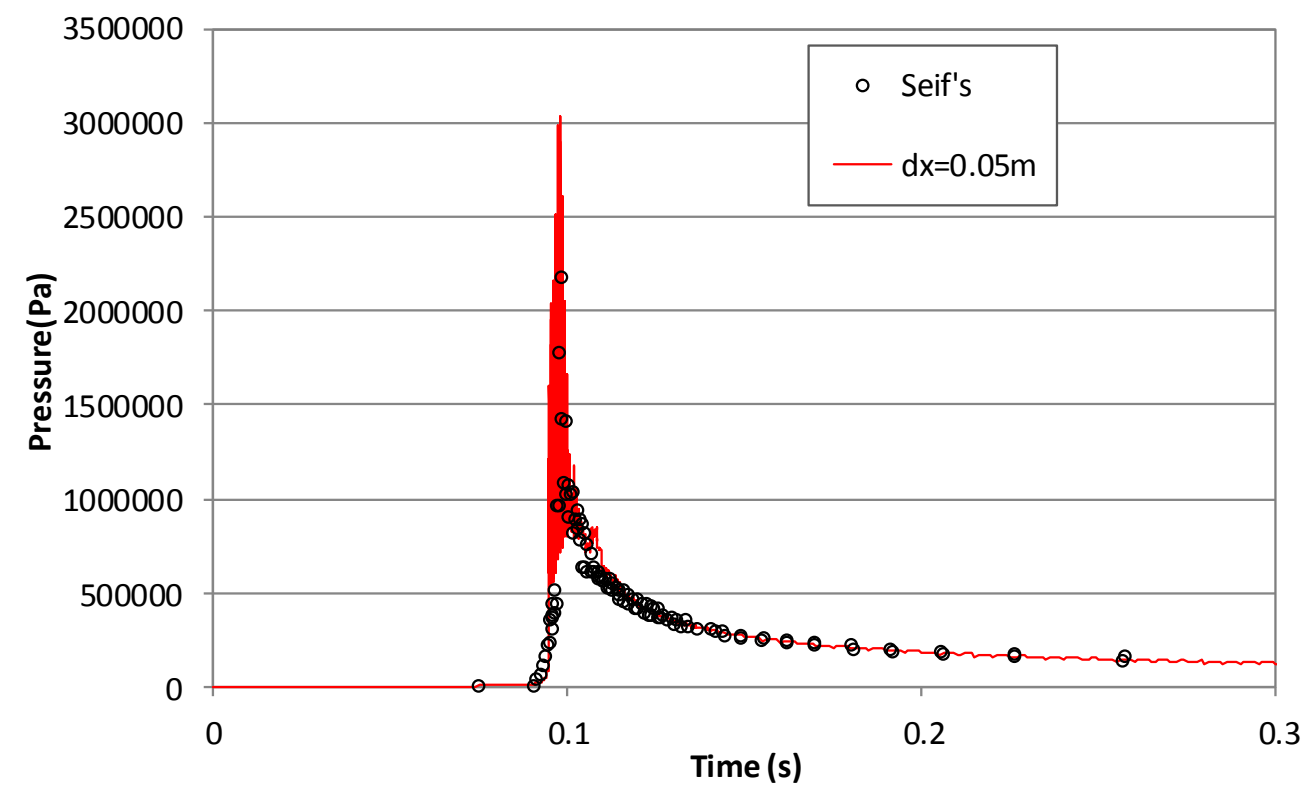

Fig.6 Pressure history at the lowest point of the cylinder 

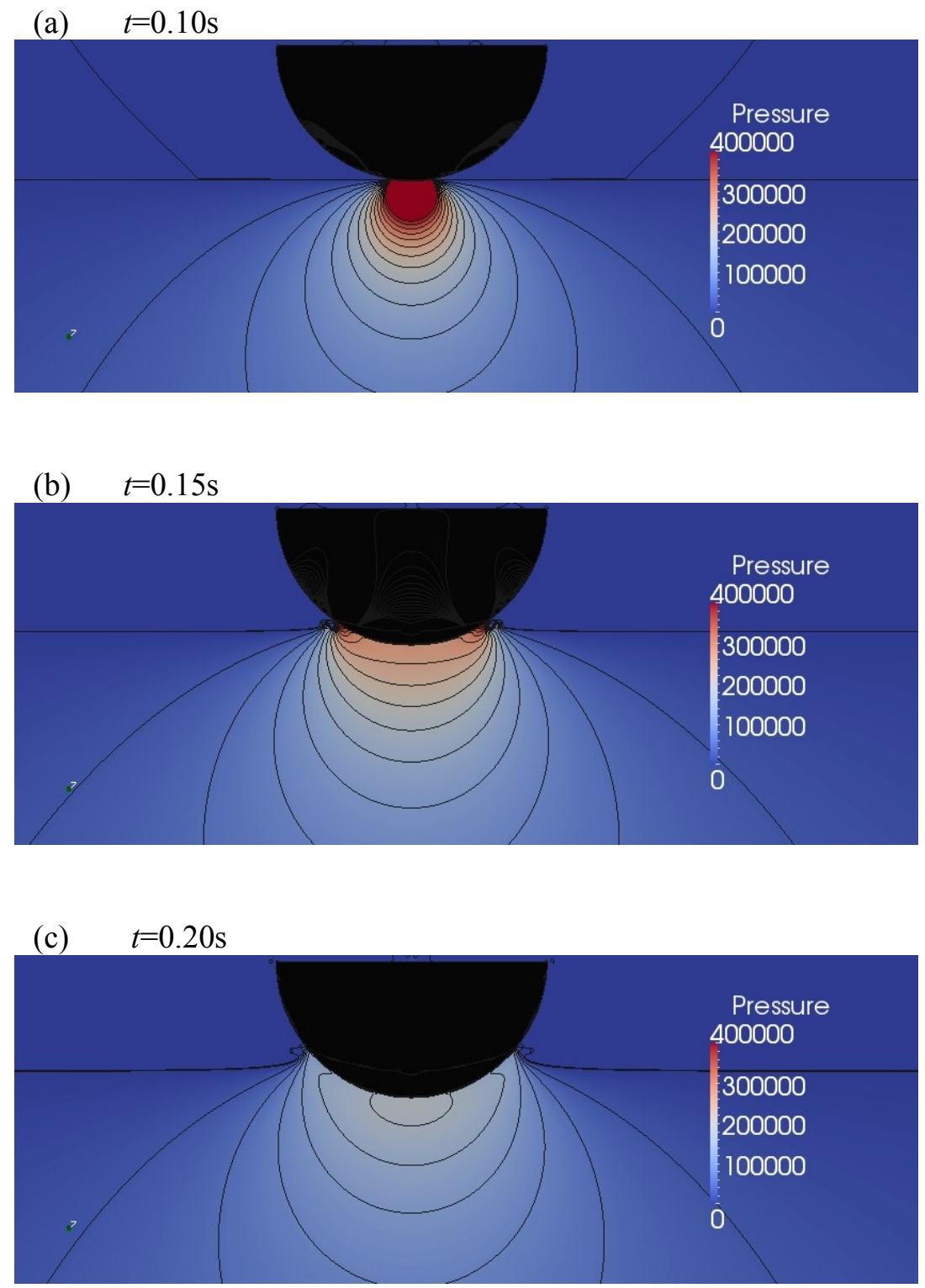

Fig.7 Pressure contours showing peak pressure values during water entry

\subsection{Vertical water entry of symmetric wedges}

To further validate our numerical model the tests conducted by Zhao et al. (1997) have been reproduced, in which a symmetric wedge with $30^{\circ}$ deadrise angle was dropped from air along a fixed rig onto the water surface. During the test the velocity and the pressure along the wetted surface of the wedge were measured. The test case has also been used as a validation benchmark for many other CFD codes (Marcer et al. 2010).

In this study, three uniform meshes are used to simulate the test case. The spatial mesh intervals are $0.02 \mathrm{~m}, 0.01 \mathrm{~m}$ and $0.005 \mathrm{~m}$ respectively. The pressure distribution along the lower surface of the wedge is plotted at $t=0.00435 \mathrm{~s}$ and $t=0.0158 \mathrm{~s}$ and compared with the experimental measurements and other CFD results in figures 8 and 9. In the figures, the pressure coefficient is defined as $c_{p}=p /\left(\frac{1}{2} \rho V(t)^{2}\right)$, in which $p$ is pressure, $\rho$ is density of water and $V$ is velocity of the moving wedge and $\Gamma$ is defined as $\Gamma=z / \int_{0}^{t} V(t) d t$, where $z$ is water depth based on the initial still water level. From 
these results, it can be seen that the maximum pressure occurs at the root of the jet, which is formed when the surface of the wedge comes in contact with water surface on either side. There are some discrepancies in pressure values among the different models. However, the results from the current model on three meshes show good spatial mesh convergence and agree reasonably well with the experimental data. A temporal convergence test for the intermediate mesh has also been carried out and the results (not shown here) are indistinguishable.

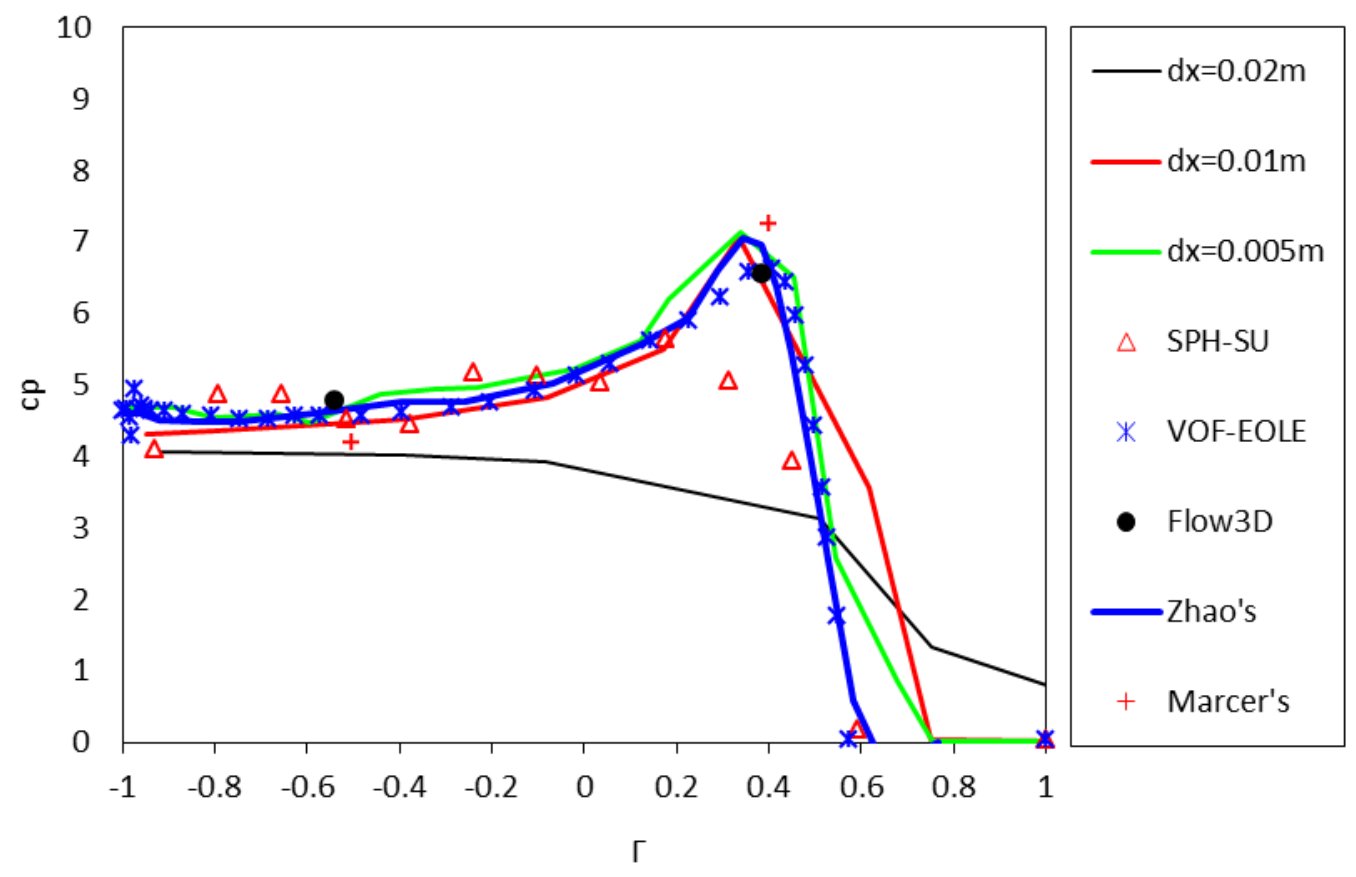

Fig. 8 Surface pressure distribution at $t=0.00435 \mathrm{~s}$

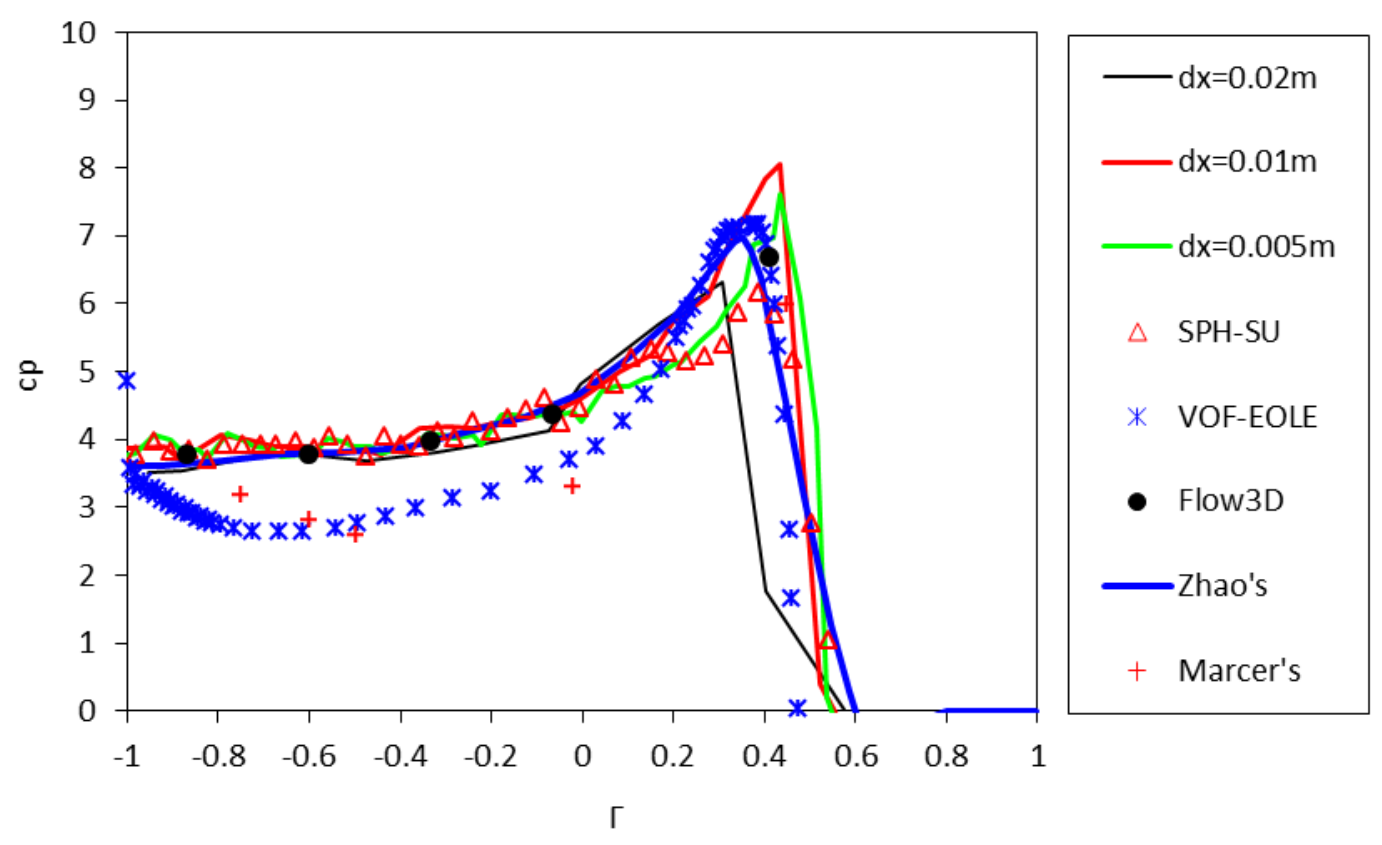

Fig.9 Surface pressure distribution at $t=0.0158 \mathrm{~s}$

\subsection{Oblique water entry of a wedge}

Oblique water entry and impact problems are important to the design of high speed planning boats but are important for any vessel articulating in several degrees of freedom in steep waves. These cases have 
attracted far less attention than vertical impact problems (Xu et al. 1998, Xu 1998; Judge 2004). Xu (1998) and $\mathrm{Xu}$ et al. (1998) categorized the flow problem of asymmetric water entry of a wedge into two types, see figure 10 for a definition sketch in which $\Theta$ is the so-called angle of heel defining the geometric asymmetry of the wedge entry. Type A is defined as the flow that moves outward from the vertex along the contour of the wedge on each side of the vertex when there is small asymmetry in the water entry. Type B flow occurs in cases of large asymmetric water entry where the flow detaches from one side of the wedge contour. Judge et al (2004) conducted both numerical and experimental tests to study initial water entry of rigid wedges for both types of flows. In this section firstly the two drop tests from Judge et al. (2004) are reproduced numerically and the results in terms of the water free surface profiles at various stages of water entry are compared with the measured experimental ones.

The first test case, which is a Type A case, is a symmetric wedge with dead-rise angle of $37^{\circ}\left(\beta=53^{\circ}\right.$, $\Theta=0^{\circ}$ ) entering initially calm water with equal horizontal velocity $U_{0}$ and vertical velocity $W_{0}$. The wedge height is $0.08 \mathrm{~m}$ and the width is $0.2 \mathrm{~m}$. In the numerical simulation, a uniform mesh with spatial step $0.002 \mathrm{~m}$ is used and the computational domain is set large enough to eliminate the influence of any boundaries on the simulation results. Figure 11 compares the calculated free surface profiles with a photograph obtained from the experimental investigation (Judge et al 2004) at similar instants in time during water entry. The main characteristics of the free surface pattern are captured well by the numerical model including the shape of the two jets formed on either side of the wedge. However, small air bubbles and foamy spray observed in the experiments are not reproduced in the simulation results due to the fact that surface tension effects have presently been ignored in the numerical model.

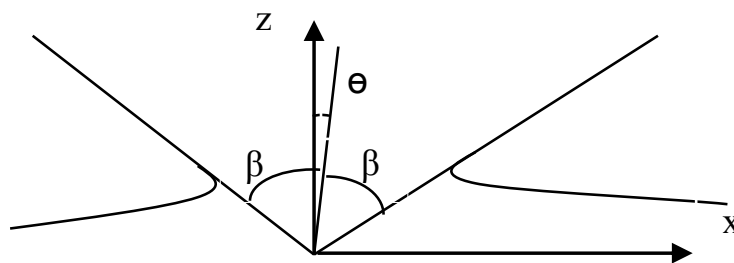

Type A

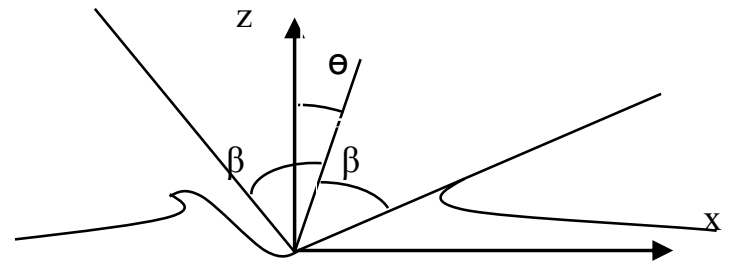

Type B

Fig. 10 Definition sketch for Type A and B flows for water entry problems

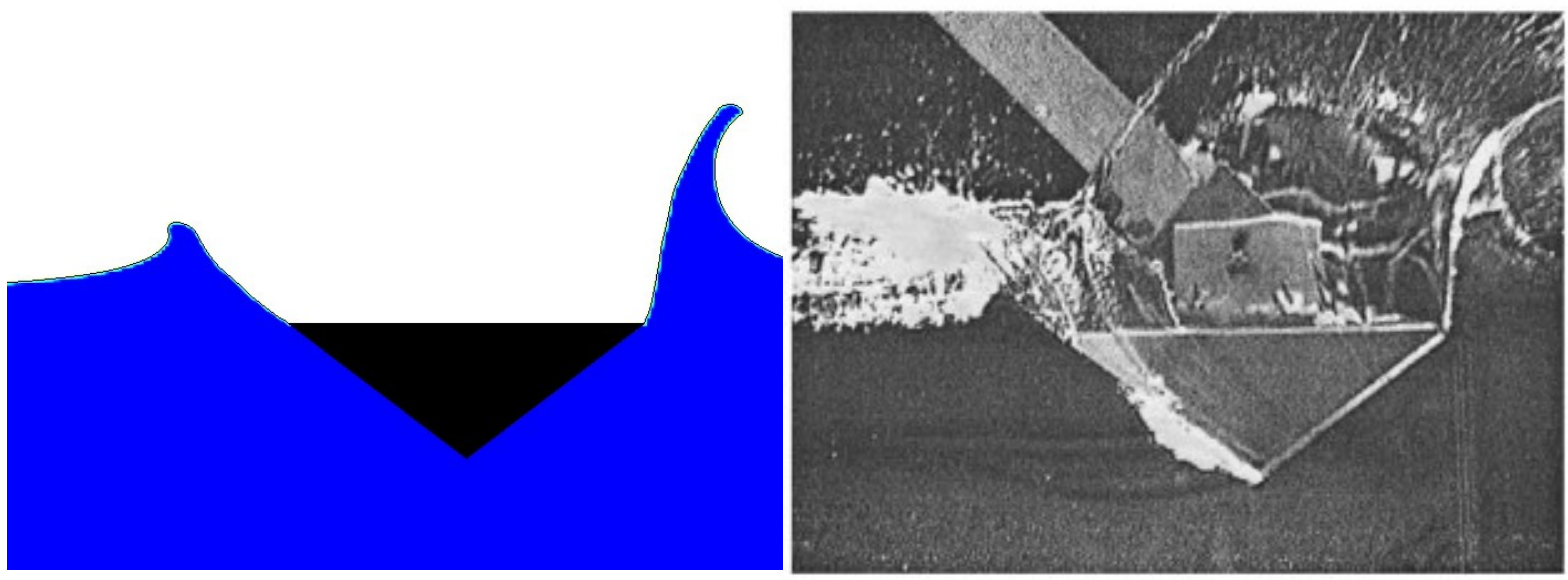

(left: present numerical results; right: cited from Judge et al. 2004)

Fig. 11 Type A flow impact under $\Theta=0^{0}$ and $\mathrm{U}_{0} / \mathrm{W}_{0}=-1.0$

The second case test is for the same wedge but with an angle of heel $\Theta=5^{0}$ and a velocity ratio $\mathrm{U}_{0} / \mathrm{W}_{0}$ $=-2.0$. This is an example of Type B flow. The difference from a Type A flow is that during entry the water free surface on the left side of the wedge separates from the wedge surface and flow ventilation occurs. Figure 12 shows the present numerical results compared with experimental photographs at various times 
during water entry. Initially the flow appears to be attached to the wedge surface and as time advances the water free surface on the left side gradually separates from the body surface and a pocket of air ventilation forms. The predicted free surface profiles resemble the experiments well. Figure 13 shows a snapshot of the pressure and velocity distribution for this type of flow.

Judge et al. (2004) examined the transition from Type A to Type B flow by increasing the angle of heel $\Theta$ at a fixed velocity ratio. In this study the present model is also used to simulate this process. Figure 14 shows the results for five $\Theta$ values from $15^{0}$ to $34^{0}$ at the velocity ratio $\mathrm{U}_{0} / \mathrm{W}_{0}=-0.75$, from which it can be seen that the flow is attached to the surface of the wedge at $\Theta=15^{\circ}$, but for $\Theta=20^{\circ}$ although the flow remains attached a separation cavity is starting to form at the vertex. The flow for these two instances is still Type A, however, for $\Theta$ values greater than $20^{\circ}$ the flow on the left-hand side detaches from the wedge surface, and the flow pattern now belongs to Type B. With the increased $\Theta$ values the flow detachment is more prominent. The numerical results agree well with the experiments.
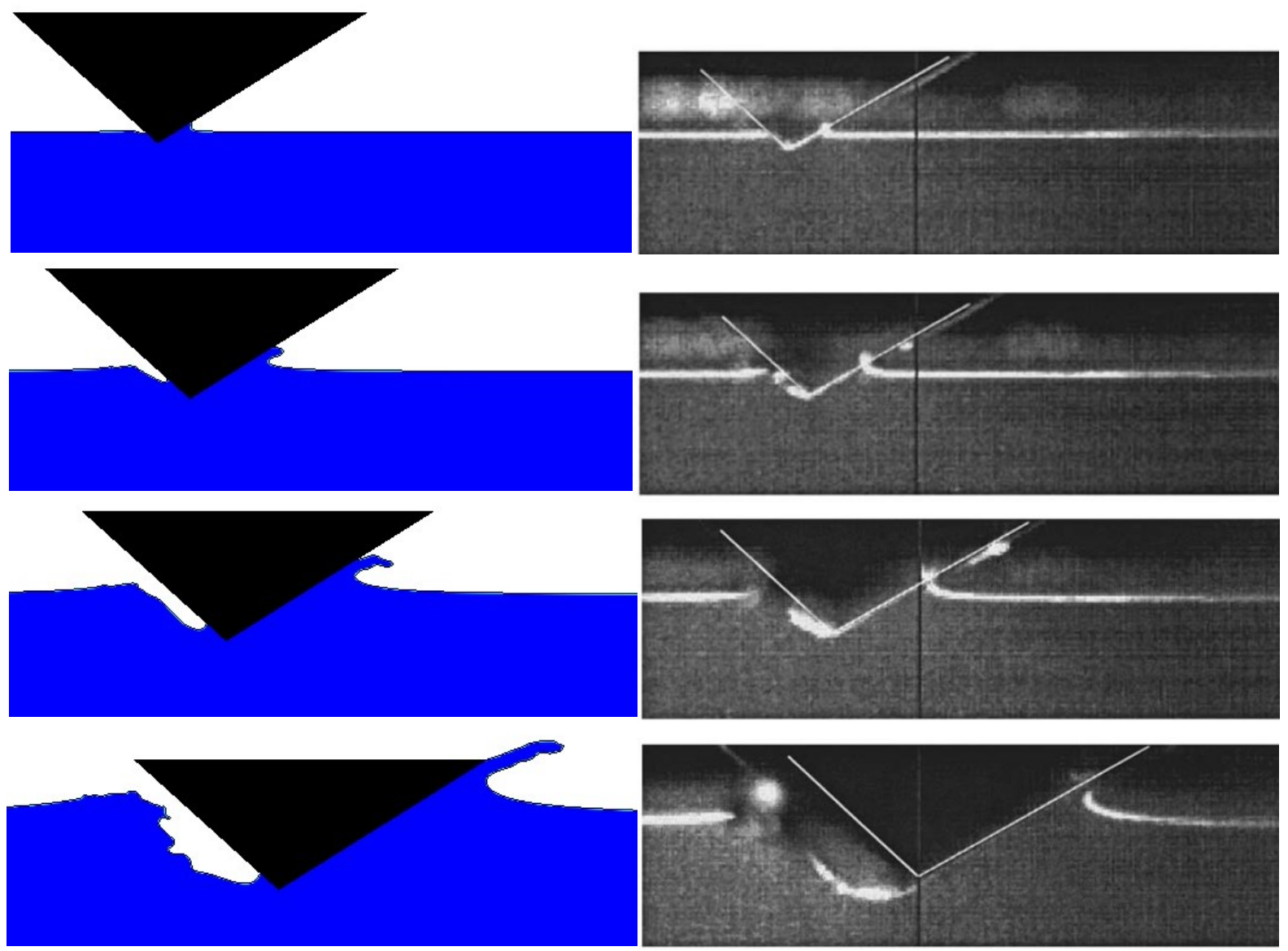

(left: present numerical results; right: cited from Judge et al. 2004)

Fig. 12 Type B flow impact with $\Theta=5^{0}$ and $\mathrm{U}_{0} / \mathrm{W}_{0}=-2.0$ 

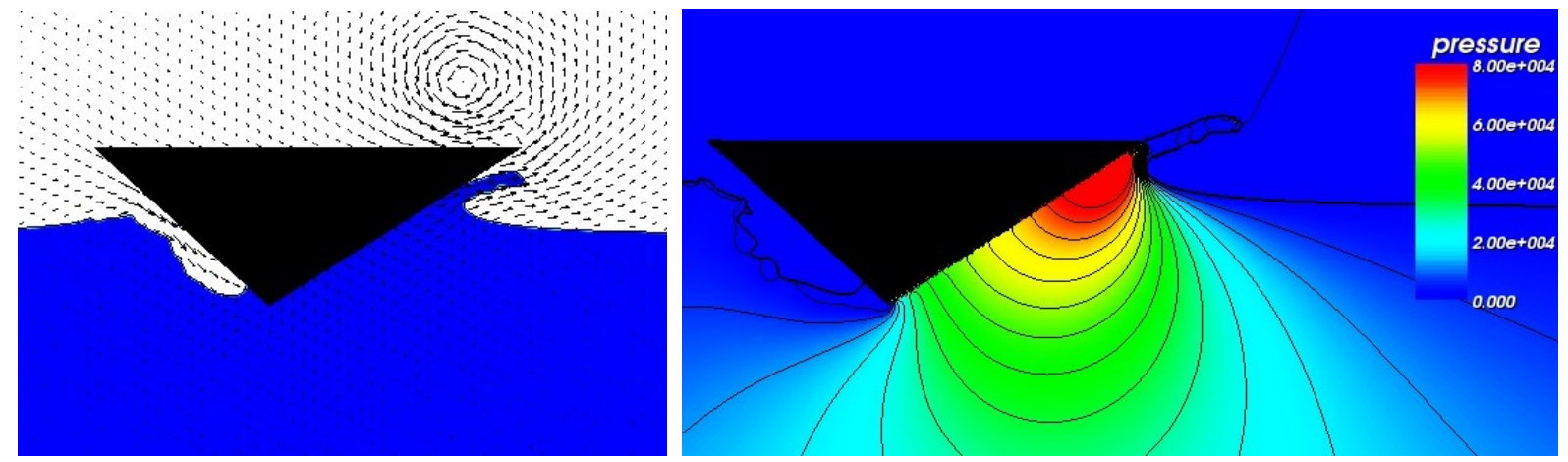

(left: Velocity field; right: Pressure distribution)

Fig. 13 Type $B$ flow impact with $\Theta=5^{0}$ and $\mathrm{U}_{0} / \mathrm{W}_{0}=-2.0$
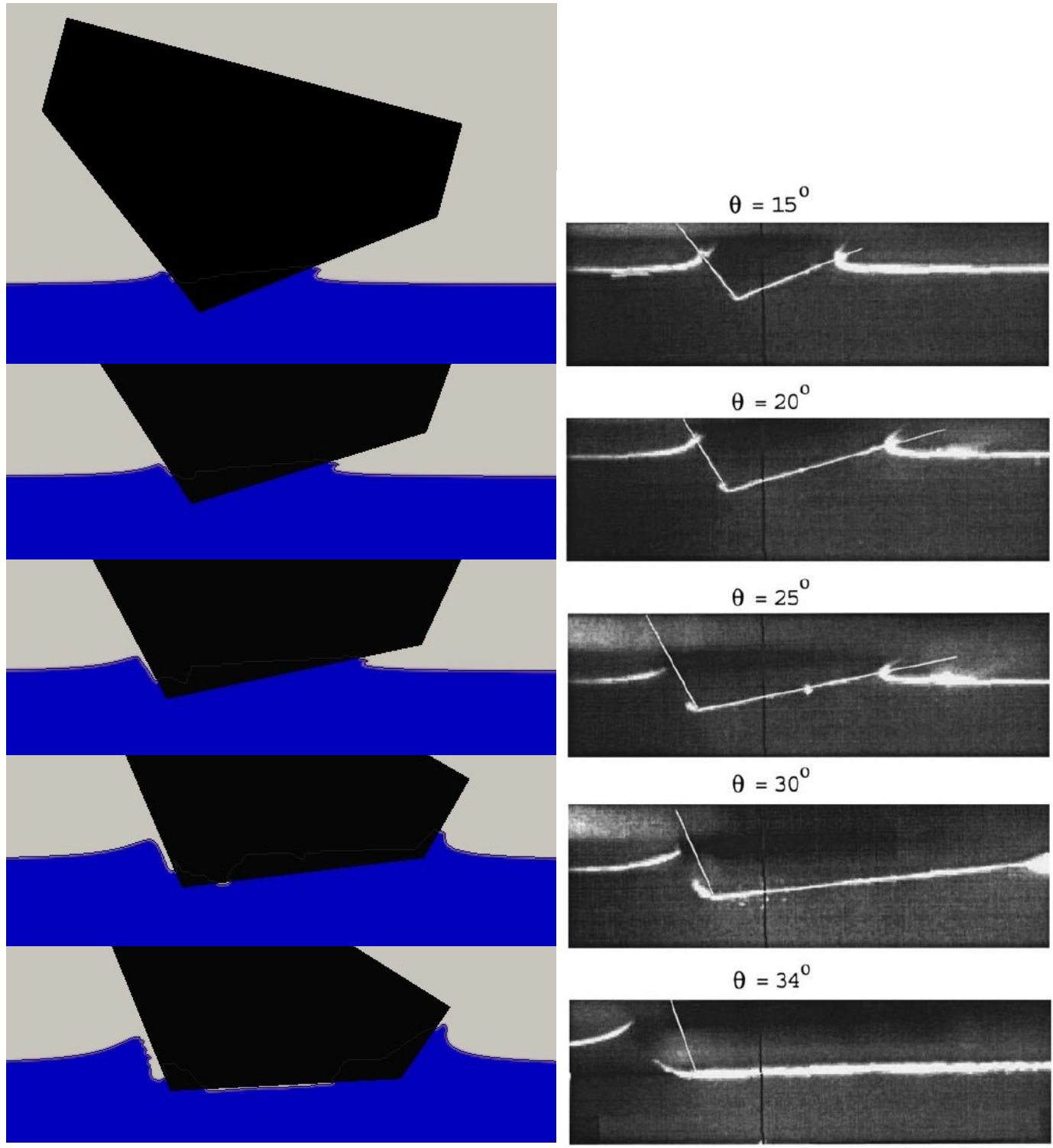

$$
\theta=34^{\circ}
$$

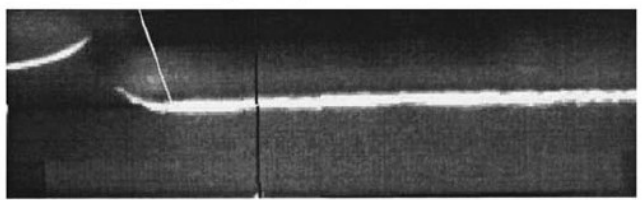

(left: present numerical results; right: cited from Judge et al. 2004)

Fig. 14 Transition from Type A to Type B impact through increasing heel angles $\left(\mathrm{U}_{0} / \mathrm{W}_{0}=-0.75\right)$ 
Next, tests of asymmetric wedges of different deadrise angles (or symmetric wedges with nonzero heel angles) with vertical and oblique water entry are simulated and the results are compared with those from a potential flow solver (Xu et al. 2008) in which gravity was omitted for all test cases. For the convenience of description, wedge angles are defined the same as that in figure 10. A parameter $\alpha$, which is defined as $\alpha=x / \int_{0}^{t} V(t) d t$, is used in the figures for the relative pressure distributions along the bottom surface of the wedge.

Table 1 Test cases for water entry of wedges

\begin{tabular}{|c|c|c|c|c|}
\hline Case No. & $\beta\left(^{\circ}\right)$ & $\Theta\left({ }^{\circ}\right)$ & $\mathrm{U}_{0}(\mathrm{~m} / \mathrm{s})$ & $\mathrm{W}_{0}(\mathrm{~m} / \mathrm{s})$ \\
\hline 1 & 60 & -10 & 0.0 & -1.0 \\
\hline 2 & 60 & -10 & 0.5 & -1.0 \\
\hline 3 & 60 & -10 & -0.5 & -1.0 \\
\hline 4 & 30 & -10 & 0.0 & -1.0 \\
\hline 5 & 30 & -10 & 0.3 & -1.0 \\
\hline 6 & 30 & -10 & -0.3 & -1.0 \\
\hline
\end{tabular}

A total of 6 test cases for two sets of wedge and a combination of three impact velocities are simulated which are listed in table 1. Figures 15 and 16 show the non-dimensional pressure distributions at various times of the initial water entry predicted by the current solver along with the results from the potential flow solver. Generally the present results agree well with those from potential flow, especially when the deadrise angles are small i.e. the cases for the wedge with $\beta=60^{\circ}$. As the deadrise angles become large as in the case for the wedge with $\beta=30^{\circ}$, the vertex of the wedge will form a sharp edge which can result in the occurrence of negative pressure locally when there is a horizontal impact velocity. From figure 16, it can be seen that the negative pressure values predicted by the potential flow theory are usually larger than those from the present model. One possible explanation for the difference is that the tips of the wedge are singular points for the potential flow and spurious flow separation can be predicted resulting in over-estimated negative pressure values for certain cases. To show this the velocity vectors and pressure contours are also plotted in figure 17 for the test cases 4,5 , and 6 at $\mathrm{t}=0.12$ seconds after water entry, from which it can be seen that no flow separation occurs for the test 4 and 5 and therefore no negative pressures are predicted by the current approach. However, when flow separation does occur as in test case 6, good agreement can be achieved between the two methods for the surface pressure distribution. 
(a) $\mathrm{U}_{0}=0.0 \mathrm{~m} / \mathrm{s}, \mathrm{W}_{0}=1.0 \mathrm{~m} / \mathrm{s}$

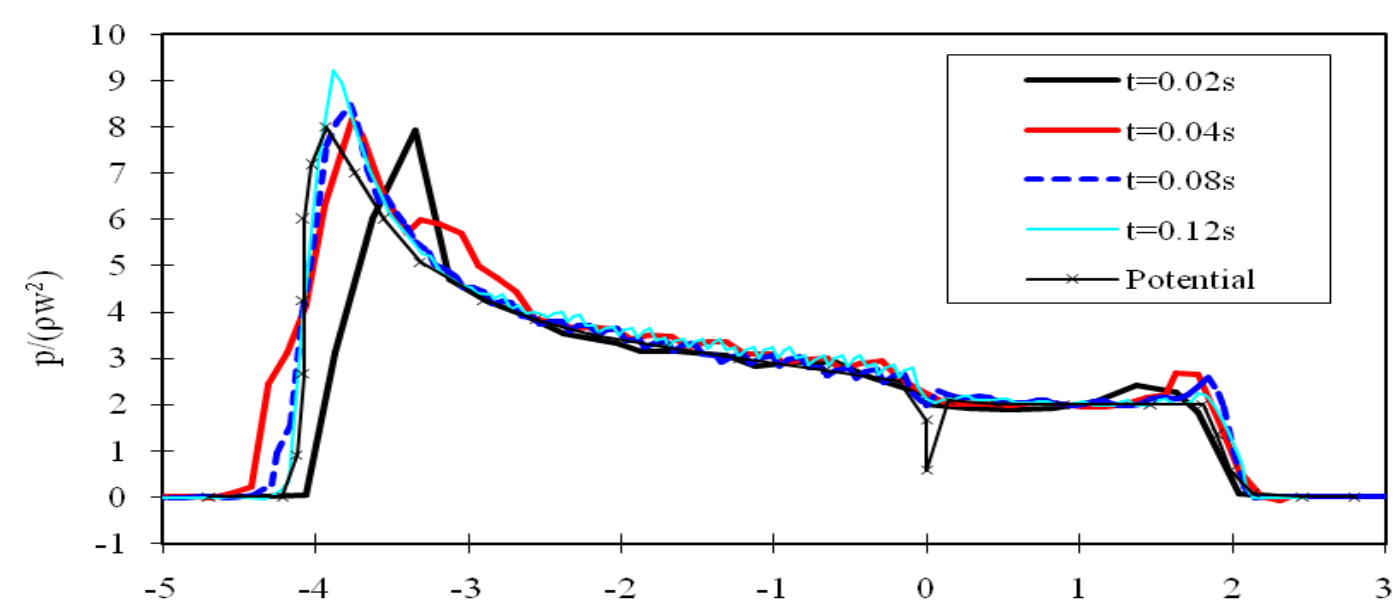

(b) $\mathrm{U}_{0}=0.5 \mathrm{~m} / \mathrm{s}, \mathrm{W}_{0}=1.0 \mathrm{~m} / \mathrm{s}$

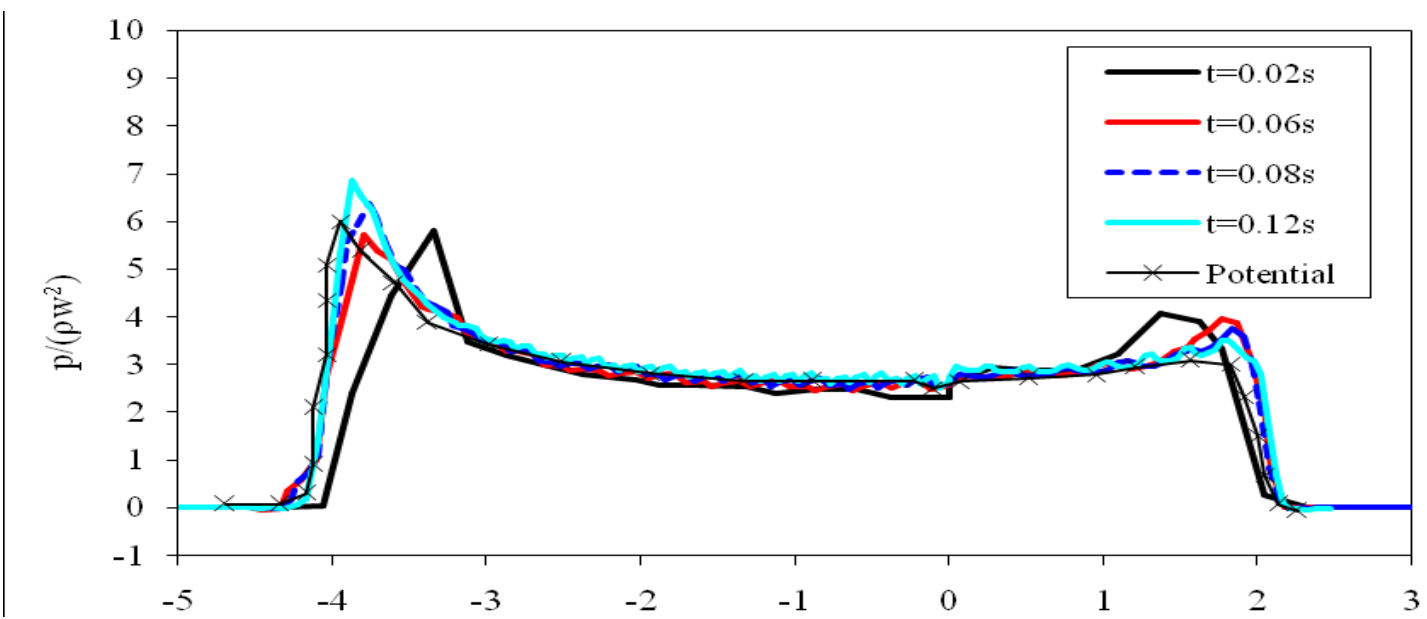

(c) $\mathrm{U}_{0}=-0.5 \mathrm{~m} / \mathrm{s}, \mathrm{W}_{0}=1.0 \mathrm{~m} / \mathrm{s}$

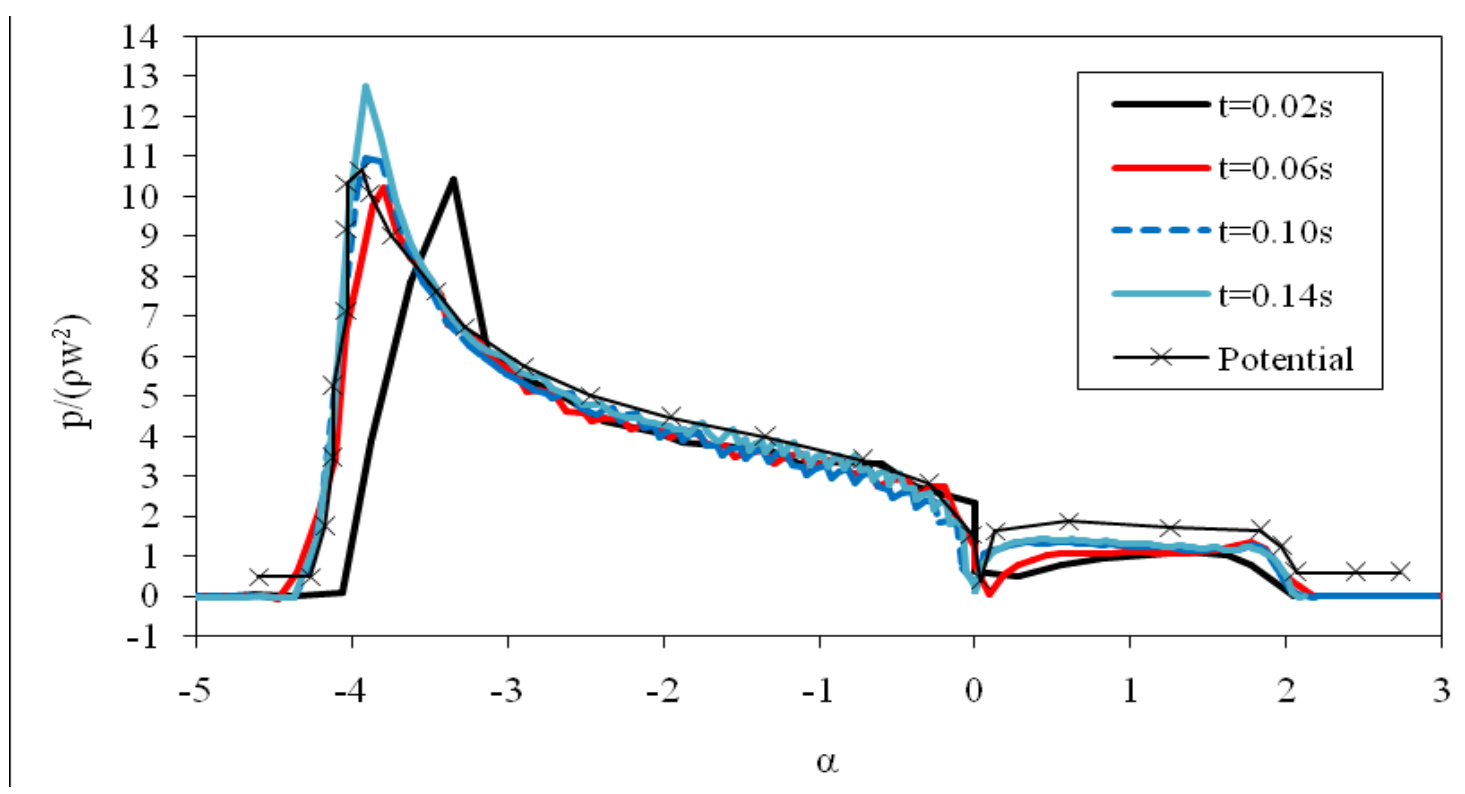

Fig. 15 Pressure distribution along the lower surface of the wedge $\beta=60^{\circ}, \Theta=-10^{\circ}$ 
(a) $\mathrm{U}_{0}=0.0 \mathrm{~m} / \mathrm{s}, \mathrm{W}_{0}=1.0 \mathrm{~m} / \mathrm{s}$

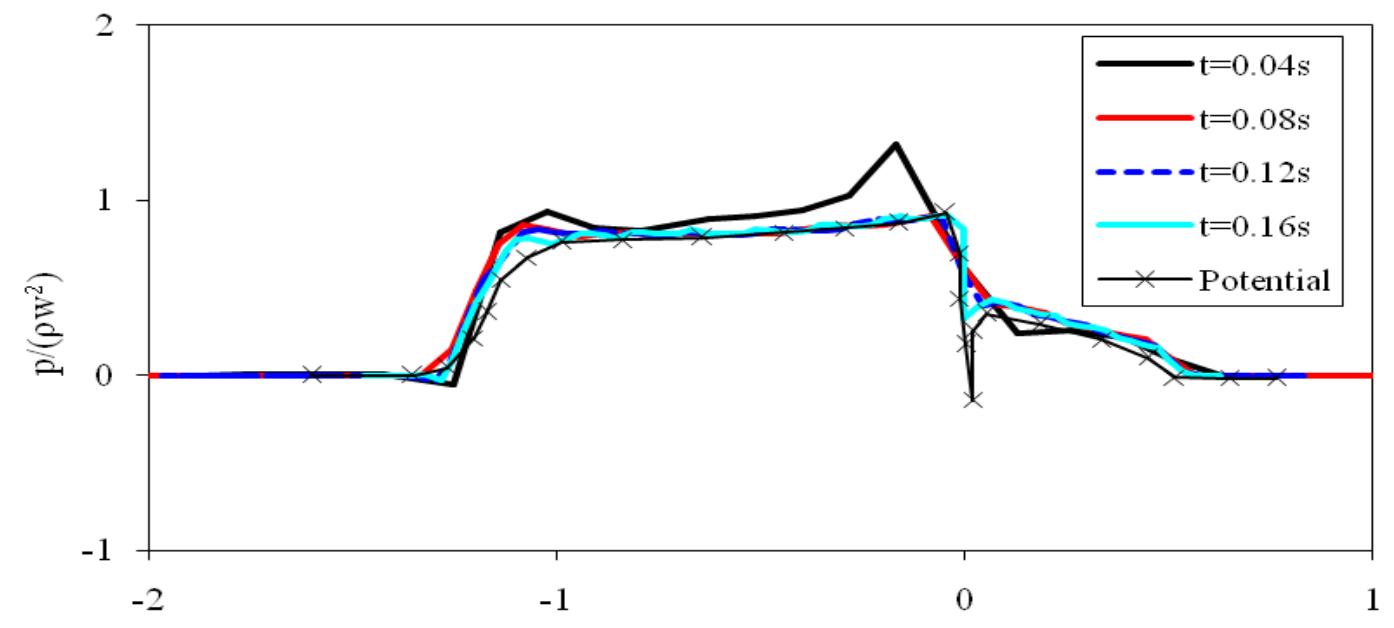

(b) $\mathrm{U}_{0}=0.3 \mathrm{~m} / \mathrm{s}, \mathrm{W}_{0}=1.0 \mathrm{~m} / \mathrm{s}$

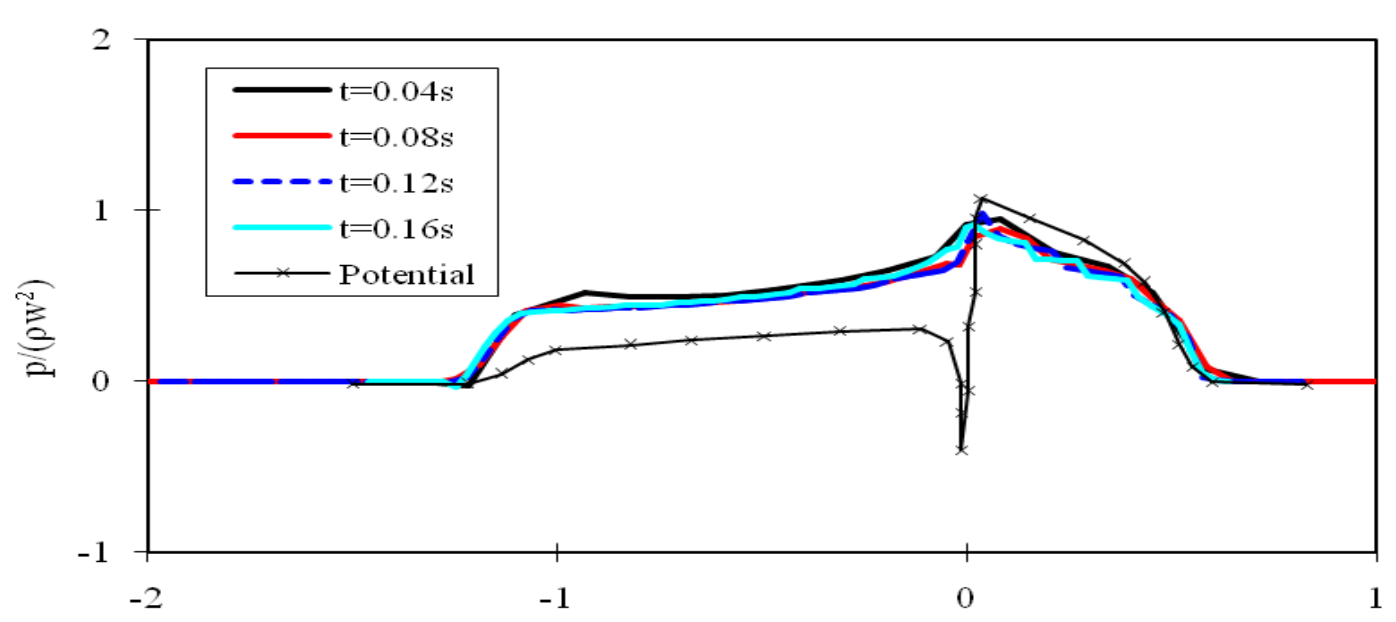

(c) $\mathrm{U}_{0}=-0.3 \mathrm{~m} / \mathrm{s}, \mathrm{W}_{0}=1.0 \mathrm{~m} / \mathrm{s}$

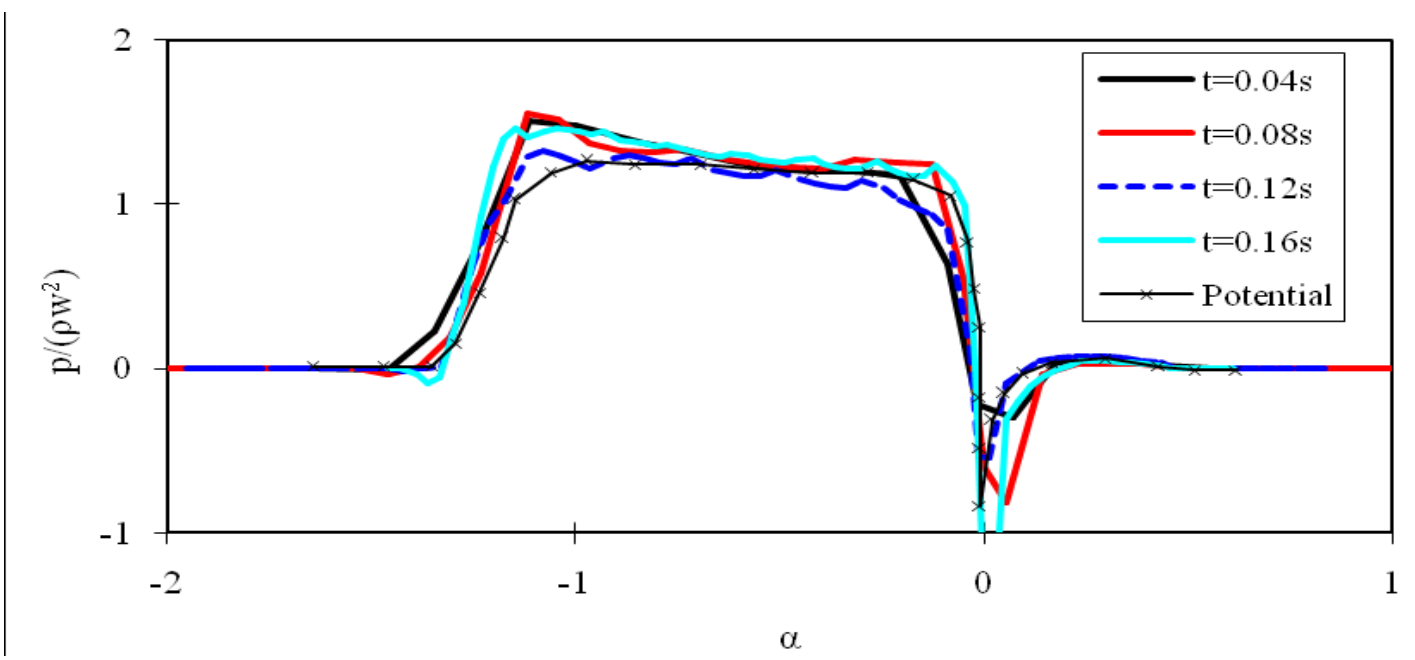

Fig.16 Pressure distribution along the lower surface of wedge $\beta=30^{\circ}, \Theta=-10^{\circ}$ 


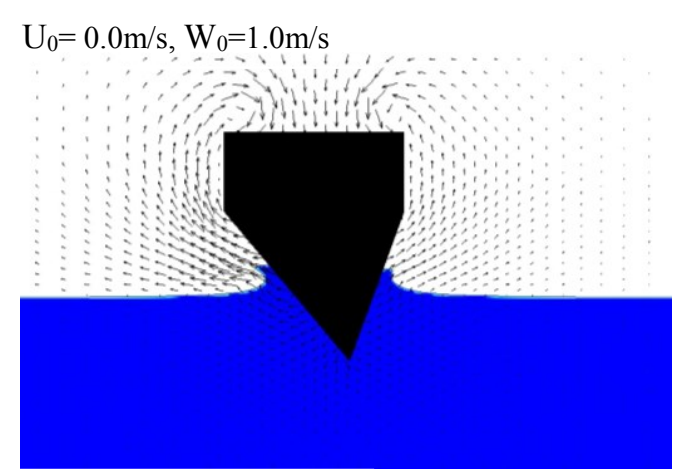

$\mathrm{U}_{0}=0.3 \mathrm{~m} / \mathrm{s}, \mathrm{W}_{0}=1.0 \mathrm{~m} / \mathrm{s}$
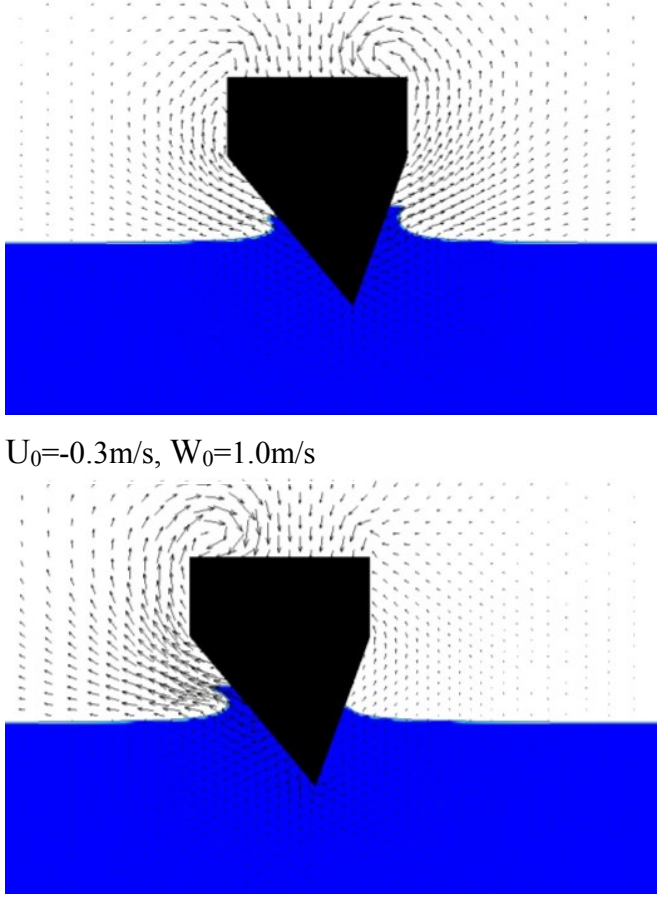
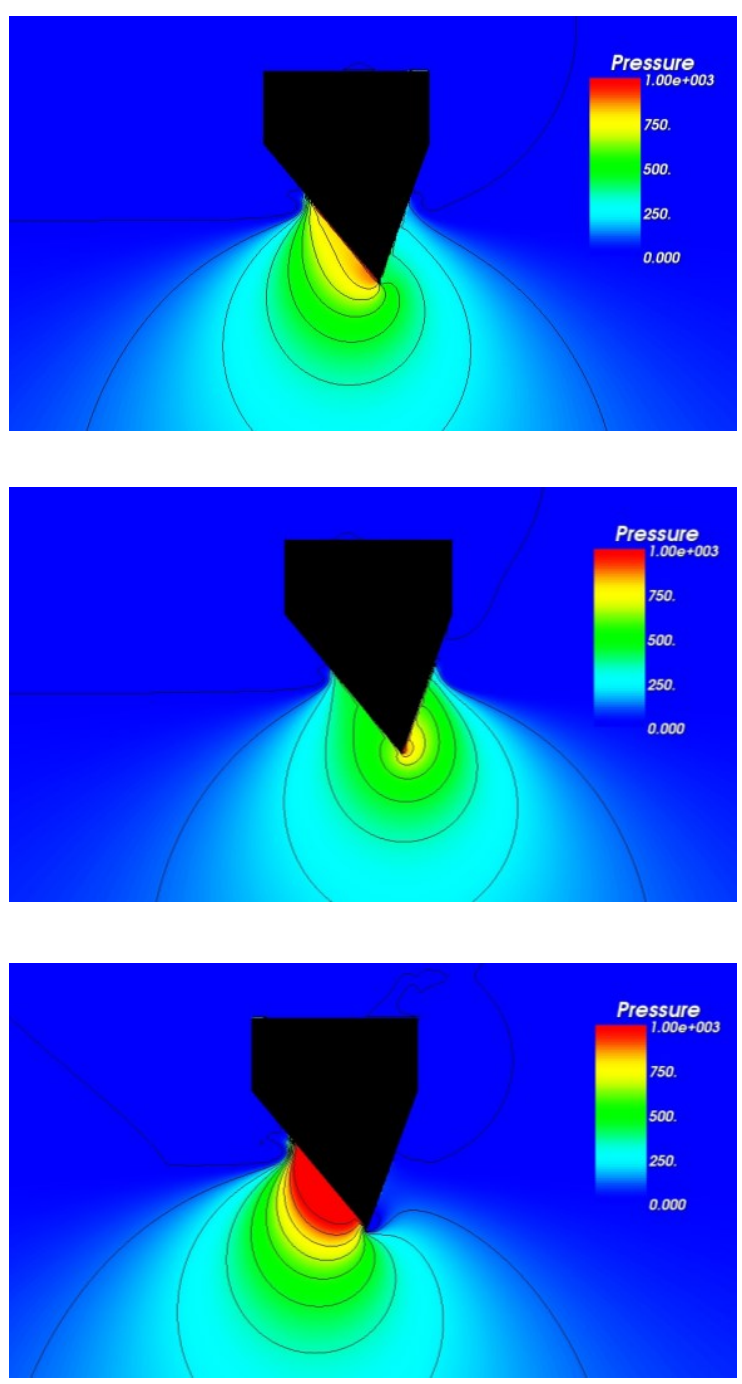

Fig. 17 Velocity vectors and pressure contours at $\mathrm{t}=0.12 \mathrm{~s}$ after water entry $\left(\beta=30^{\circ}, \Theta=-10^{\circ}\right)$

\section{Conclusions}

In this paper a newly developed free surface solver has been applied to study the flow problem of vertical and oblique water entry of solid objects of various shapes and configurations. The numerical model is based on a two-fluid incompressible NS formulation using the level set method to capture water free surface and partial cell combined with local relative stationary method to describe moving body. Numerical simulations of water entry of a semi-circular cylinder, symmetric and asymmetric wedges are presented and the results compare well with other experimental data or numerical results. For oblique water entry of wedges, both flow types with water surface attached to both sides of the wedge or detached from one side have been reproduced showing the generic and versatile nature of the method. The simulation results also highlight the fact that the solutions from the potential theory can sometimes give erroneous pressure values at the vertex of asymmetric wedges for certain cases with large deadrise angles due to the inherent limitations in the model. 


\section{References}

1. Arai M., Cheng L.Y., Inoue Y. (1994) A computing method for the analysis of water impact of arbitrary shaped bodies. J. Society Naval Architects Japan 176.

2. Armand J.L., Cointe R. (1987) Hydrodynamic impact analysis of a cylinder. J. Offshore Mech. Artic Engng., 9, 237-243.

3. Campbell I.M.C., Weinberg P.A. (1980) Measurement of parameters affecting slamming. Wolfson Unit, University of Southampton, Report No. 440.

4. Chen Y.G., Price W.G. (2009) Numerical simulation of liquid sloshing in a partially filled container with inclusion of compressibility effects. Physics of fluids, 21.

5. Cointe R. (1987) Two dimensionnal fluid-solid impact. J. Offshore Mech. Artic Engng., 111, 109-114.

6. Cointe R. (1991) Free-surface flows close to a surface-piercing body. In: T. Miloh (ed.), Mathematical Approaches in Hydrodynamics. Philadelphia: SIAM.

7. Dobrovol'skaya Z.N. (1969) On some problem of similarity flow of fluid with a free surface. J. Appl. Mech., 36, 805-829.

8. Enright D., Fedkiw R., Ferziger J., Mitchell I. (2002) A hybrid particle level set method for improved interface capturing. Journal of Computational Physics, 183, 83-116

9. Enright D., Losasso F., Fedkiw R. (2005) A fast and accurate semi-Lagrangian particle level set method. Computers \& Structures, 83, 479-490.

10. Fraenkel L.E. Keady G. (2004) On the entry of a wedge into water: The thin wedge and an all-purpose boundarylayer equation. J. Engng Maths, 48, 219-252.

11. Fraenkel L. E., McLeod J. B. (1997) Some results for the entry of a blunt wedge into water. Phil. Trans. R. Soc. Lond. A, 355, 523-535.

12. Gao F., Ingram D.M., Causon D.M., Mingham C.G. (2007) The development of a Cartesian cut cell method for incompressible viscous flows. Int. J. Numer. Meth. Fluids, 54, 1033-1053.

13. Greenhow M. (1987) Wedge entry into initially calm water, Applied Ocean Research, 9, 4, 214-223.

14. Greenhow M., Yanbao L. (1987) Added masses for circular cylinders near or penetrating fluid boundaries-review and application to water-entry, exit and slamming. J. Ocean Engng., 14, 325-348.

15. Gu H.B., Causon D.M., Mingham C.G., Qian L. (2009) A fast-marching semi-lagrangian level set method for free surface flows. ISOPE.

16. Gu H.B., Causon D.M., Mingham C.G., Qian L. (2010) High resolution computation of free surface flows using a level set approach. ISOPE.

17. Guendelman E., Selle A., Losasso F., Fedkiw R. (2005) Coupling water and smoke to thin deformable and rigid shells. ACM Transactions on Graphics (TOG) - Proceedings of ACM SIGGRAPH 2005 TOG Homepage, July, 24(3), 973-981.

18. Harlow F., Welch J. (1965) Numerical calculation of time-dependent viscous incompressible flow. Phys. of Fluids, $8,2182-2189$.

19. Hirano Y., Miura K. (1970) Water impact accelerations of axially symmetric bodies. J. of Spacecraft, 7(6), 762764.

20. Hirt C.W., Nichols B.D. (1981) Volume of fluid (VOF) method for the dynamics of free boundaries. Journal of Computational Physics, 39, 201-225.

21. Howison S.D., Ockendon J.R., Wilson S.K. (1991) Incompressible water-entry problems at small deadrise angles. J. Fluid Mech., 222, 215-230.

22. Jiang G.S., Peng D.P. (2000) Weighted ENO schemes for Hamilton-Jacobi equations. SIAM J. SCI. COMPUT, 21(6), 2126-2143.

23. Jiang G.S., Wu C.C. (1999) A high-order WENO finite difference scheme for the equations of ideal magnetohydrodynamics. Journal of Computational Physics, 150(2), 561-594.

24. Judge C., Troesch A., Perlin M. (2004) Initial water impact of a wedge at vertical and oblique angles. Journal of Engineering Mathematics, 48, 279-303.

25. Kelecy F.J., Pletcher R.H. (1997) The development of a free surface capturing approach for multidimensional free surface flows in closed containers. Journal of Computational Physics, 138, 939-980.

26. Korobkin A.A. (1996) Water impact problems in ship hydrodynamics. In: M. Ohkusu (ed.), Water Impact Problems in Ship Hydrodynamics. Southampton: Computational Mechanics Publications, 323-371.

27. Korobkin A.A. (1997) Asymptotic theory of liquid-solid impact. Phil. Trans.: Math. Phys. Eng. Sci. 35, 507-522.

28. Korobkin A.A., Peregrine H. (2000) Energy distribution during liquid-solid impact. J. Fluid Mech., 418, 157-181.

29. Korobkin A.A., Khabakhpasheva T.I. (2006) Impact on the boundary of a compressible two-layer fluid. Fluid Dynamics, 41(2), 263-277

30. Korobkin A.A. (2006) Two-dimensional problem of the impact of a vertical wall on a layer of a partially aerated liquid. Journal of Applied Mechanics and Technical Physics, 47(5), 643-653.

31. Koshizuka S., Oka Y. (1995) Development of a particle method for calculating fragmentation of incompressible viscous fluid', Proc. US-Japan Joint Seminar: A Multidisciplinary Int. Seminar Intense Multiphase Interactions, 145-158.

32. Lin P. (2007) A fixed-grid model for simulation of a moving body in free surface flows. Computers \& Fluids, 136, 549-561 
33. Lin P. (1998) Numerical modeling of breaking waves. A dissertation presented to the Faculty of the Graduate School of Cornell University.

34. $\mathrm{Lu} \mathrm{CH}, \mathrm{He}$ YS, Wu GX (2000) Coupled analysis of nonlinear interaction between fluid and structure during impact. Journal of Fluids and Structures, 14, 127-146.

35. Mackie A.G. (1962) A linearized theory of the water entry problem. Q. J. Mech. Appl. Maths, 2, 137-151.

36. Marcer R., Berhault C., de Jouëtte C., Moirod N., Shen L. (2010) Validation of CFD codes for slamming. European Conference on Computational Fluid Dynamics, ECCOMAS CFD, J. C. F. Pereira and A. Sequeira (Eds), Lisbon, Portugal, 14-17 June

37. Mei X., Liu Y. Yue D.K.P. (1999) On the water impact of general two-dimensional sections. Appl. Ocean Res., 21, $1-15$.

38. Mulder W., Osher S., Sethian J. (1992) Computing interface motion in compressible gas dynamics. Journal of Computational Physics, 100(2), 209-228.

39. Muzaferija S., Peric M., Sames P., Schellin T. (1998) A two-fluid Navier-Stokes solver to simulate water entry. In: Proc. 22nd Symp. Naval Hydrodyn. Washington D.C.: National Academy Press, 638-651.

40. Oger G., Doring M., Ferrant P., Allesandrini B. (2005) 2D SPH simulations of wedge water entry, YCPH.

41. Osher S., Sethian J.A. (1988) Fronts propagating with curvature-dependent speed: Algorithms based on HamiltonJacobi formulations. Journal of Computational Physics, 79, 12-49

42. Pan D., Chang C.H. (2000) The capture of free surface in incompressible multi-fluid flows. Int. J. Num. Meth. Fluids, 33, 203-222

43. Qian L., Causon D.M., Ingram D.M., Mingham C.G. (2003) A Cartesian cut cell two-fluid solver for hydraulic flow problems. ASCE Journal of Hydraulic Engineering, 129(9), 688-696.

44. Qian L., Causon D.M., Mingham C.G., Ingram D.M. (2006) A free-surface capturing method for two fluid flows with moving bodies. Proc. R. Soc. A, 462, 21-42.

45. Seif S.M, Mousaviraad M.S., Saddathosseini H.S., Bertram V. (2005) Numerical modelling of 2-D water impact in one degree of freedom. Síntesis Tecnológica, 2(2) 79-83.

46. Sethian J. (1998) Fast marching methods. Dept. of Mathematics, University of California, Berkeley.

47. Shao S., Ji C., Graham D.I., Reeve D.E., James P.W., Chadwick A.J. (2006) Simulation of wave overtopping by an incompressible SPH model. Coastal engineering 53(9), 723-735.

48. Shu C.W., Osher S. (1989) Efficient implementation of essentially non-oscillatory shock-capturing schemes. Journal of Computational Physics, 83(1), 32-78.

49. Strain J. (1999) Semi-Lagrangian methods for level set equations. Journal of Computational Physics, 151(2), 498533.

50. Strain J. (2000) A fast modular semi-Lagrangian method for moving interfaces. Journal of Computational Physics, $161,512-536$.

51. Toyama Y. (1996) Flat plate approximation in the three-dimensional slamming (in Japanese). Journal of the Society of Naval Architects of Japan, 179, 271-279.

52. Ubbink O. (1997) Numerical prediction of two fluid systems with sharp interfaces. Thesis, Department of mechanical engineering, Imperial college of science, technology \& medicine January.

53. Von Kármán Th.(1929) The impact on seaplane floats during landing. NACA TN 321.

54. Wagner H. (1932) Über stoß und gleitvorgänge an der oberläche von flüssigkeiten. ZAMM 12, 193-215.

55. Wu GX (2007) Fluid impact on a solid boundary. Journal of Fluids and Structures, 23:755-765.

56. Wu GX (2007) Two dimensional liquid column and liquid droplet impact on a solid wedge. Quarterly Journal of Mechanics and Appllied Mathematic, 60, 497-511.

57. Wu GX, Sun H, He YS (2004) Numerical simulation and experimental study of water entry of a wedge in free fall motion. Journal of Fluids and Structures, 19, 277-289.

58. Xu GD, Duan WY, Wu GX (2008) Numerical simulation of oblique water entry of an asymmetrical wedge. Ocean Engineering, 35, 1597-1603.

59. Xu L. (1998) A theory for asymmetrical vessel impact and steady planning. PhD thesis, University of Michigan, Ann Arbor, Michigan 128.

60. Xu L., Troesch A.W., Vorus W.S. (1998) Asymmetric vessel impact and planning hydrodynamics. J. Ship Res. 42, 187-198.

61. Yang J., Stern F. (2009) Sharp interface immersed-boundary/level set method for wave-body interactions. Journal of Computational Physics, 228, 6590-6616.

62. Youngs D.L. (1982) Time - dependent multi - material flow with large fluid distortion. In: Numerical methods for fluid dynamics, K. W. Morton and M. J. Bianes (Eds.), Academic Press, New York, 273.

63. Zhao R., Faltinsen O.M. (1993) Water entry of two-dimensional bodies. Journal of Fluid Mechanics, 246, 593-612.

64. Zhao R., Faltinsen O., Aarsnes J. (1997) Water entry of arbitrary two-dimensional sections with and without separation. In Proc. Twenty-first Symp. on Naval Hydrodynamics, 408-423. 